Journal reference for this post-print...

Journal of Optics A: Pure \& Applied Optics 10 (2008) 115007 (16pp) (IOP PUBLISHING) 


\title{
Relativistic Hermite polynomials and Lorentz beams
}

\author{
A. Torre ${ }^{(a)}$ \\ ENEA-FIM-FIS-MAT Tecnologie Fisiche e Nuovi Materiali, \\ via E. Fermi 45, 00044 Frascati (Rome), Italy \\ W.A.B. Evans \\ School of Physical Sciences, University of Kent, \\ Canterbury, CT2 7NH, England \\ O. El Gawhary ${ }^{(b)}$ \\ Via G. Matteotti 189, 02032 Fara in Sabina (Ri), Italy \\ S. Severini \\ Centro Interforze Studi per le Applicazioni Militari \\ Via della Bigattiera 10, 56010 San Piero a Grado (Pi), Italy
}

The link between the relativistic Hermite polynomials and the Lorentz beams is evidenced. That suggests introducing new optical fields. The paraxial propagation properties of such fields are studied in detail. They are finally put in relation with the so-called Weber-Hermite beams, emerged within a certain class of general solutions of the $1 \mathrm{D}$ paraxial wave equation in Cartesian coordinates as a result of a recent re-analysis of such an equation.

Keywords: Hermite polynomials; Gaussian optical beams; Paraxial wave equation PACS: $02.30 . \mathrm{Gp} ; 45.25 .-\mathrm{p}$

\section{Introduction}

The wide spread of experimental investigations about the possibility of producing optical beams with specific features useful for applications, for instance, in optical trapping, optical tweezers, image processing, microlithography and so on, yields a strong stimulus for analytical investigations on the paraxial wave equation in free space, aimed at identifying new solutions, which may suggest new kinds of realizable optical fields. Correspondingly, investigations may also involve specific optical fields, possibly emerged from experimental observations, whose propagation in free space is then analyzed with the intent of producing, if possible, a closed-form expression for it.

This was the case indeed of the Lorentz beams. Recently introduced in [1] on the basis of the experimental observations reported in $[2,3]$, their paraxial propagation properties have been found to be expressible through an analytical form and accordingly studied in detail in the quoted paper.

Interestingly, such beams have been more recently framed within the context of a certain class of general separation-variable based solutions of the 1D paraxial wave equation, as presented in [4] and later in [5] on the basis of different approaches.

On the other hand, it is always intriguing to identify unsuspected relations between apparently unrelated fields of physics. In this vein, we wish to show the link - to the authors' knowledge still undiscussed in the literature - between the Lorentz beams and

\footnotetext{
${ }^{a}$ Corresponding author. E-mail: torre@frascati.enea.it; Fax:+39-06-94005334

${ }^{b}$ Now with the Optics Research Group, Dept. of Imaging Science and Technology, Delft University of Technology, Lorentzweg 1, 2628 CJ Delft, The Netherlands
} 
the relativistic Hermite polynomials (RHP). The latter have been introduced less than two decades ago within the context of the dynamics of the relativistic quantum harmonic oscillator [6], and later recognized to be tightly connected with the Gegenbauer polynomials (GP) $[7,8]$, and hence more in general with the Jacobi polynomials [9].

In this regard, we recall that the analogy between quantum mechanics and wave optics is indeed well established; it emerges through the correspondence principle from the variational principle-based analogy between Hamiltonian mechanics and geometrical optics, the light wavelength $2 \pi / k$ playing the role of the Planck constant $h$. The well known analogy between light optics and charged-particle optics is a primary example of this, which analogy seems to be extendible from the "classical" context to the "quantum" one.

So, in Sect. 2, after briefly reviewing the definition and the basic properties of the RHPs, whose relation with the Lorentz - or, more in general, multi-Lorentzian - beams resides in the relative Rodrigues representation, we suggest, as new optical fields, waveforms shaping as the product of multi-Lorentzian factors times RHPs of suited "relativistic" parameter $N$. We name them elegant Hermite-(or, Gegenbauer-)Lorentzian beams (EHLB or EGLB). It is also noted that the waveforms introduced relate to the $1 \mathrm{D}$ version of the functions constructed in [10] within the framework of Clifford analysis as mother wavelets for a higher dimensional wavelet transform. In Sect. 3 we investigate the paraxial propagation properties of the suggested optical fields, detailing also the role of the parameter $N$, which we refer to as multiplicity parameter. Also, in virtue of the aforementioned relation of the Lorentz beams with a general solution of the 1D paraxial wave equation, we will frame the EHL beams within the same paraxial wave equation related context, clarifying their link to what have been referred to as Weber-Hermite beams in [5]. Conclusive notes are given in Sect. 4, where we suggest a radial version of the introduced EHL beams by virtue of that relativistic Laguerre polynomials have been introduced [11] - and later related to the Jacobi polynomials as well [9]. So, one may think of elegant Laguerre-(or, Jacobi) Lorentzian beams, which could presumably be put in relation with the general solution of the paraxial wave equation in cylindrical coordinates, involving Whittaker's first function $M_{\kappa, \mu}[12,13]$. A formal account of the possibility of introducing the fractional order version of the EHL beams - following the analysis in [14]- closes the paper.

\section{The relativistic Hermite polynomials}

The RHPs $H_{n}^{(N)}(\xi)$ have been introduced in [6] as the polynomial component of the wave function of the quantum relativistic harmonic oscillator. In the asymptotic (nonrelativistic) limit, $c \rightarrow \infty$, they become the ordinary Hermite polynomials (HP), which enter indeed as polynomial components in the quantum non-relativistic harmonic oscillator eigenfunctions. Explicitly, the RHPs have been originally worked out in the form $[6]$

$H_{n}^{(N)}(\xi)=\frac{n !}{2^{n}} \frac{\Gamma\left(N+\frac{1}{2}\right)}{N^{\frac{n}{2}}} \frac{\Gamma(2 N+n)}{\Gamma(2 N)} \sum_{j=0}^{\left[\frac{n}{2}\right]} \frac{(-)^{j}}{j !(n-2 j) !} \frac{1}{\Gamma\left(N+j+\frac{1}{2}\right)}\left(\frac{2 \xi}{\sqrt{N}}\right)^{n-2 j},-\infty<\xi<+\infty$

for non negative real value of the dimensionless parameter $N$. Defined by the ratio of the particle energy $m c^{2}$ to the oscillator energy quantum $\hbar \omega_{0}: N=\frac{m c^{2}}{\hbar \omega_{0}}$, it signals the "relativistic" character of the polynomials (2.1), which evidently turn into the ordinary Hermite polynomials $H_{n}(\xi)$ in the limit $N \rightarrow \infty$. 
As mentioned earlier, the RHPs relate to the GPs $C_{n}^{N}$ [15]. In fact, since

$$
\Gamma\left(N+j+\frac{1}{2}\right)=\frac{(-)^{j} \Gamma\left(N+\frac{1}{2}\right) \Gamma\left(\frac{1}{2}-N\right)}{\Gamma\left(\frac{1}{2}-N-j\right)},
$$

we may see that

$$
H_{n}^{(N)}(\xi)=(-i)^{n} \frac{n !}{2^{n} N^{\frac{n}{2}}} \frac{\Gamma\left(\frac{1}{2}-N-n\right)}{\Gamma\left(\frac{1}{2}-N\right)} \frac{\Gamma(2 N+n)}{\Gamma(2 N)} C_{n}^{\frac{1}{2}-N-n}\left(i \frac{\xi}{\sqrt{N}}\right)
$$

which, on account of the relation

$$
C_{n}^{\nu-n}(x)=(-2)^{n}\left(x^{2}-1\right)^{\frac{n}{2}}\left(\begin{array}{c}
n-\nu \\
n
\end{array}\right)\left(\begin{array}{c}
n-2 \nu \\
n
\end{array}\right)^{-1} C_{n}^{\frac{1}{2}-\nu}\left(\frac{x}{\sqrt{x^{2}-1}}\right)
$$

yields the further expression relating the RHPs to the GPs:

$$
H_{n}^{(N)}(\xi)=\frac{n !}{N^{\frac{n}{2}}}\left(1+\frac{\xi^{2}}{N}\right)^{\frac{n}{2}} C_{n}^{N}\left(\frac{\xi}{\sqrt{N}\left(1+\frac{\xi^{2}}{N}\right)^{\frac{1}{2}}}\right),
$$

alternative proofs of which have been given in [7]-[9].

"Relativistic" versions of Laguerre and Jacobi polynomials have been introduced as well, all being later recognized as Jacobi polynomials of suitable different arguments [9]. The interest in the $H_{n}^{(N)}$ 's here is only motivated by their relation with the Lorentz beams, which then naturally suggests to frame the $H_{n}^{(N)}$ 's within an optical context.

So, in the following we briefly summarize the basic properties of the RHPs, which evidently follow from the relation (2.3).

The polynomials (2.1) are even or odd according to the evenness of the index $n$, since $H_{n}^{(N)}(-\xi)=(-)^{n} H_{n}^{(N)}(\xi)$. Furthermore, they obey the recurrence relations

$$
\begin{aligned}
& H_{n+1}^{(N)}(\xi)=2\left(1+\frac{n}{N}\right) \xi H_{n}^{(N)}(\xi)-\left(1+\frac{\xi^{2}}{N}\right) \frac{d}{d \xi} H_{n}^{(N)}(\xi), \\
& \frac{d}{d \xi} H_{n}^{(N)}(\xi)=\frac{n}{N}(2 N+n-1) H_{n-1}^{(N)}(\xi),
\end{aligned}
$$

which amount to the differential equation

$$
\left\{\left(1+\frac{\xi^{2}}{N}\right) \frac{d^{2}}{d \xi^{2}}-2\left(1+\frac{n-1}{N}\right) \xi \frac{d}{d \xi}+2 n\left(1+\frac{n-1}{2 N}\right)\right\} H_{n}^{(N)}(\xi)=0 .
$$

In the limit $N \rightarrow \infty$ the above turns into the well-known differential equation obeyed by the HPs [15], and accordingly it yields an operator form representation of the RHPs, which parallels that of the HPs. Indeed, following the technique of solution suggested in [16], we rewrite Eq. (2.5) in terms of the scale transform generator $\widehat{S}=\xi \frac{d}{d \xi}$, i.e.

$$
\left[\frac{d^{2}}{d \xi^{2}}+\frac{1}{N}(\widehat{S}-2 N-n+1)(\widehat{S}-n)\right] y(\xi)=0,
$$

and map the relevant solution in the space of monomials $\xi^{\lambda}$, where $\widehat{S}$ is diagonal, taking

$$
y(\xi)=Y_{\lambda, N, n}\left\{\sum_{j=0}^{\infty}(-)^{j}\left[\frac{N}{\mathcal{L}(\widehat{S}, N, n)} \frac{d^{2}}{d \xi^{2}}\right]^{j}\right\} \xi^{\lambda},
$$


with $\mathcal{L}(\widehat{S}, N, n)=(\widehat{S}-2 N-n+1)(\widehat{S}-n)$, under the condition that $\lambda$ be chosen in order to satisfy the equation

$$
\mathcal{L}(\widehat{S}, N, n) \xi^{\lambda}=(\widehat{S}-2 N-n+1)(\widehat{S}-n) \xi^{\lambda}=0 .
$$

The above gives $\lambda=n$ and $\lambda=2 N+n-1$. The former leads to the polynomial expression of the RHPs, which then can be written as

$$
H_{n}^{(N)}(\xi)=\frac{\Gamma(2 N+n)}{N^{n} \Gamma(2 N)}\left\{\sum_{j=0}^{\infty}(-)^{j}\left[\frac{N}{\mathcal{L}(\widehat{S}, N, n)} \frac{d^{2}}{d \xi^{2}}\right]^{j}\right\} \xi^{n},
$$

choosing the constant $Y_{\lambda, N, n}$ to match the definition in (2.1). It can be verified in fact that the above results in the polynomials in (2.1), since

$$
(-)^{j}\left[\frac{N}{\mathcal{L}(\widehat{S}, N, n)} \frac{d^{2}}{d \xi^{2}}\right]^{j} \xi^{n}=\frac{(-)^{j}}{j !}\left(\frac{N}{4}\right)^{j} \frac{\Gamma\left(N+\frac{1}{2}\right)}{\Gamma\left(N+j+\frac{1}{2}\right)} \frac{d^{2 j}}{d \xi^{2 j}} \xi^{n},
$$

on account of the operator rule

$$
[\widehat{O}(x)]^{-1} f(x)=\int_{0}^{\infty} d t e^{-\widehat{O}(x) t} f(x),
$$

and the commutation relation $\left[\widehat{S}, \frac{d^{2}}{d \xi^{2}}\right]=-2 \frac{d^{2}}{d \xi^{2}}$.

In addition, as earlier noted, (2.9) yields an interesting operatorial expression for the RHPs, which parallels that holding for the GPs [16] and the HPs $[17,16]$. In fact, after some algebra we end up with

$$
H_{n}^{(N)}(\xi)=\frac{\Gamma(2 N+n)}{N^{n} \Gamma(2 N)} e^{\frac{N}{2(\widehat{S}-2 N-n+1)} \frac{d^{2}}{d \xi^{2}}} \xi^{n} .
$$

In the non relativistic limit $N \rightarrow \infty$ we recover the well known exponential operator form for the HPs $[17,16]$ :

$$
H_{n}(\xi)=2^{n} e^{-\frac{1}{4} \frac{d^{2}}{d \xi^{2}}} \xi^{n} .
$$

Apart from their formal elegance and unifying valence, operatorial representations like (2.10) and (2.11) may usefully be exploited in several contexts. Within the optical propagation (or, more in general, evolutionary) related context, for instance, the representation (2.11) allows us to obtain the closed-form expression for the propagation of the elegant and standard Hermite-Gaussian beams only through operator methods.

Finally, the orthogonality relation for the RHPs can be stated as $[6,8]$

$$
\int_{-\infty}^{\infty}\left(1+\frac{\xi^{2}}{N}\right)^{-\left(N+\frac{n+m}{2}+1\right)} H_{n}^{(N)}(\xi) H_{m}^{(N)}(\xi) d \xi=\pi n ! \frac{2^{1-2 N}}{N^{n-\frac{1}{2}}} \frac{\Gamma(2 N+n)}{(N+n) \Gamma(N)^{2}} \delta_{n, m},
$$

and the Rodrigues formula as

$$
H_{n}^{(N)}(\xi)=(-)^{n}\left(1+\frac{\xi^{2}}{N}\right)^{N+n} \frac{d^{n}}{d \xi^{n}}\left(1+\frac{\xi^{2}}{N}\right)^{-N} .
$$

Relations (2.12) and (2.13) will be the starting point of our considerations. The orthogonality relation suggests in fact to define the orthonormal functions $[6,8]$

$$
\varphi_{n, S}^{(N)}(\xi)=\mathcal{N}_{n, N}^{(S)} H_{n}^{(N)}(\xi)\left(1+\frac{\xi^{2}}{N}\right)^{-\frac{N+n+1}{2}},
$$


the relevant normalization factor being $\mathcal{N}_{n, N}^{(S)}=2^{N-\frac{1}{2}} \Gamma(N)\left[\frac{N^{n-\frac{1}{2}}(N+n)}{\pi n ! \Gamma(2 N+n)}\right]^{\frac{1}{2}}$, whereas the Rodrigues formula signals the convenience of introducing the functions

$$
\varphi_{n, E}^{(N)}(\xi)=\mathcal{N}_{n, N}^{(E)} H_{n}^{(N)}(\xi)\left(1+\frac{\xi^{2}}{N}\right)^{-(N+n)}
$$

with $\mathcal{N}_{n, N}^{(E)}=2^{N-\frac{1}{2}} \Gamma(N)\left[\frac{N^{n-\frac{1}{2}} \Gamma\left(N+n+\frac{1}{2}\right)}{\sqrt{\pi} \Gamma(N+n) \Gamma\left(2 N+n-\frac{1}{2}\right) \Gamma\left(n+\frac{1}{2}\right)}\right]^{\frac{1}{2}}$ (see the Appendix for details). The meaning of the labels $\mathrm{S}$ and $\mathrm{E}$ will became clear in the following.

Both functions involve the RHPs which are then modulated by the Lorentzian function $\frac{1}{1+\frac{\xi^{2}}{N}}$ raised to an appropriate power.

In this connection, we recall that, on the basis of the experimental observations illustrated in $[2,3]$, Lorentz beams, i.e. beams described by fields displaying a spatial dependence at the plane $z=0$ of the type $\frac{1}{1+x^{2}}$, have been introduced in [1], where their propagation has been also studied in detail. Additionally, they have been framed within the context of the general separation-variable based solution of the 1D paraxial wave equation, as recently discussed in $[4,5]$. A further analysis concerning products of Lorentz beams - referred to as super-Lorentzian beams - has been developed in [18].

In the light of this, it is rather natural to try to envisage an "optical interpretation" of the functions $\varphi_{n, S}^{(N)}$ and $\varphi_{n, E}^{(N)}$.

Indeed, the obvious limit relation $\left(1+\frac{\xi^{2}}{N}\right)^{-N} \underset{N \rightarrow \infty}{\rightarrow} e^{-\xi^{2}}$ suggests that the $\varphi_{n, E}^{(N)}$, s be considered as the "relativistic" limit of the elegant Hermite Gaussian (EHG) beams $\varphi_{n, E}$, which shapes as $\varphi_{n, E}(\xi) \propto H_{n}(\xi) e^{-\xi^{2}}$, whereas the $\varphi_{n, S}^{(N)}$, s could be regarded as corresponding to the standard Hermite Gaussian (SHG) beams $\varphi_{n, S}$, which in turn, with a different scaling of the 'space' variable $\xi$, shapes as $\varphi_{n, S}(\xi) \propto H_{n}(\xi) e^{-\frac{\xi^{2}}{2}}$. The aforeintroduced labels $\mathrm{S}$ and $\mathrm{E}$ signify indeed the correspondence of the functions (2.14) and (2.15) respectively with the standard and elegant Gaussian beams, the latter being viewed as the "non-relativistic" limit of the former.

However, behind formal speculations, it seems evident that only the $\varphi_{n, E}^{(N)}$, s are amenable for an optical implementation on account of the aforementioned experimental observations $[2,3]$ and the Rodrigues formula (2.13). In fact, as suggested by $(2.13)$, the $\varphi_{n, E}^{(N)}$, s could be optically produced by firstly Fourier transforming what we may call the multi-Lorentzian beam $\left(1+\frac{\xi^{2}}{N}\right)^{-N}$, with $N$ integer ${ }^{(1)}$. The resulting beam could then be allowed to propagate through an optical element of transmittance $T \propto(-i \xi)^{n}$ and finally Fourier transformed back. Neglecting the possible effects of the limited aperture of the optical transmittance, we assume the resulting beam represent just the optical realization of the function $\varphi_{n, E}^{(N)}$. We might refer to it as the (elegant) Hermite-Lorentzian (or GegenbauerLorentzian, HL or GL) beam of order $n$ and (integer) parameter $N$, which acts as a sort

\footnotetext{
${ }^{1}$ Throughout we will deal with the Lorentz-like factor $\left(1+\frac{\xi^{2}}{N}\right)^{-N}$, since it plainly gives the Gaussian $e^{-\xi^{2}}$ in the limit $N \rightarrow \infty$. Evidently, different normalization of the variable $\xi$ might be adopted. Also, as noted earlier, products of Lorentz beams of the type $\prod_{j=1}^{M} \frac{1}{1+\frac{\xi^{2}}{w_{j}^{2}}}$ have been introduced in [18] and referred to as super-Lorentzian-beams. Even though the factor $\left(1+\frac{\xi^{2}}{N}\right)^{-N}$ resembles in a sense the aforementioned products when setting there $M=N$ and $w_{j}=w, j=1, . ., M$, we prefer to refer to it as multi-Lorentzian function, since the relative limit for $N \rightarrow \infty$ produces the Gaussian $e^{-\xi^{2}}$ instead of the exponential $e^{-\xi^{m}}$, $m>2$, expected for a super-Gaussian beam.
} 

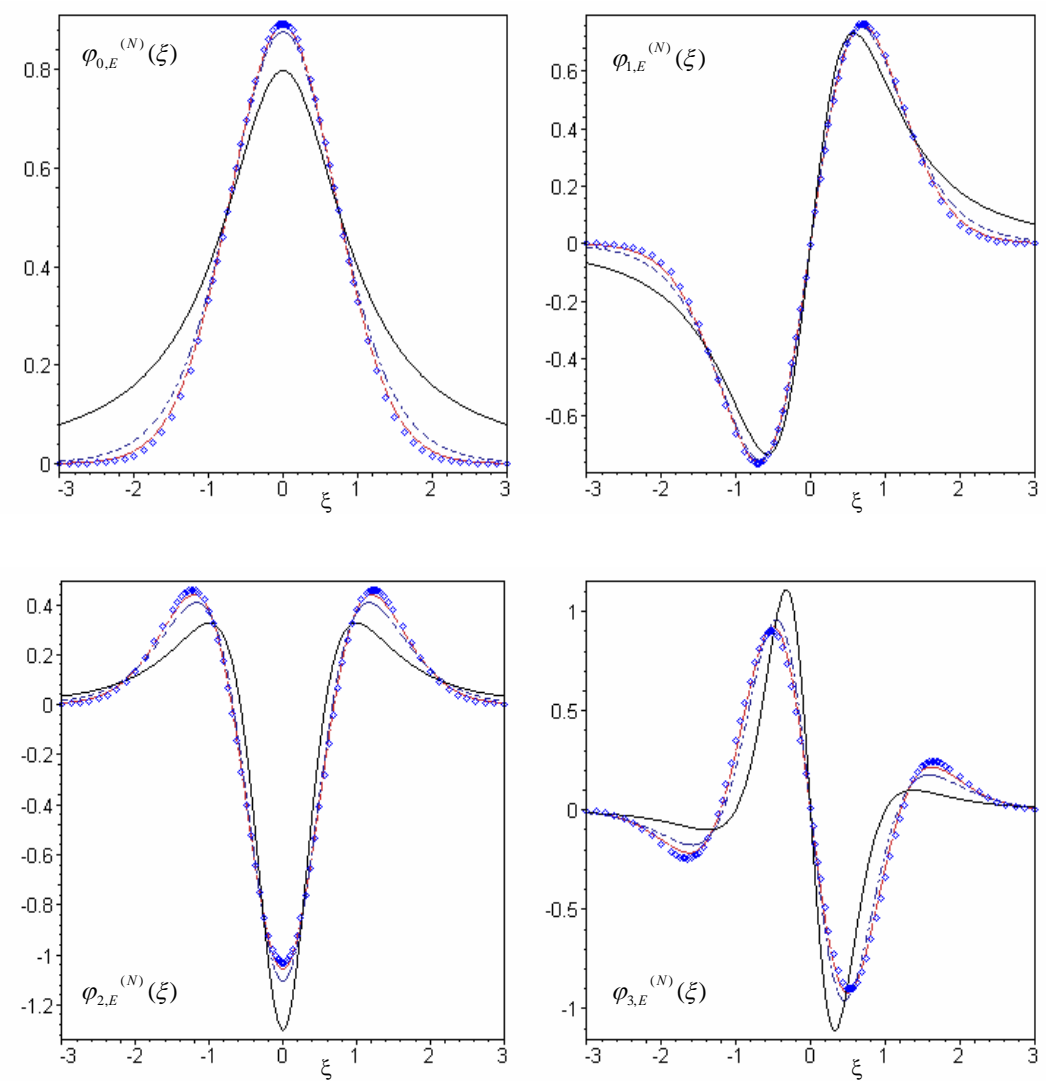

Figure 2.1: $\xi$-profiles of the EHL functions $\varphi_{n, E}^{(N)}$ 's for $n=0,1,2,3$ and $N=1$ (solid line), $N=5$ (dash-dotted line), $N=15$ (dashed line). The profiles of the corresponding EHG functions $\varphi_{n, E}^{(\infty)}$ for each value of $n$ are also plotted, marked by the $\square$ 's.

of trait d'union between the Lorentz and the Gaussian beams. Also, $N$ determines the multiplicity of the poles $\xi_{0}= \pm i \sqrt{N}$ in the complex plane of the multi-Lorentzian factor $\left(1+\frac{\xi^{2}}{N}\right)^{-N}$ entering the $\varphi_{n, E}^{(N)}$ s, which is of interest when evaluating integral involving such functions, like for instance the Fresnel or the Fourier integral (the latter being, indeed, a specific case of the former). So, we may refer to $N$ as the multiplicity parameter.

Noteworthy, functions related to the RHPs - precisely given as $\left(1+\frac{\xi^{2}}{N}\right)^{-N} H_{n}^{(N-n)}(\xi)=$ $(-)^{n} \frac{d^{n}}{d \xi^{n}}\left(1+\frac{\xi^{2}}{N}\right)^{-N+n}$ for $n<N$ and $\xi$ replaced by its $m$-dimensional radial counterpart have been introduced in [10] within the context of the Clifford analysis. Indeed, the polynomials there introduced, referred to as Clifford-Gegenbauer polynomials, just turn into the RHPs for $m=1$. So, the aforementioned functions have been shown to be appropriate building blocks for new specific wavelet kernel functions within a higher dimensional wavelet transform. We will resort to them in the concluding notes in Sec. 4.

A visualization of the behavior of the $\varphi_{n, E}^{(N)}$, s for some values of $n$ and $N$ is offered by the plots in Fig. 2.1, where for comparison's purposes for each $n$ the corresponding EHG functions are also plotted, marked by the $\square$ 's. We recall that the normalized EHG functions writes as $\varphi_{n, E}^{(\infty)}(\xi)=\frac{1}{\sqrt{2^{n-\frac{1}{2}} \Gamma\left(n+\frac{1}{2}\right)}} H_{n}(\xi) e^{-\xi^{2}}$. It emerges from the graphs that 
for the values of $n$ here considered the EHL functions nearly approach the EHG ones for $N>15$. Evidently, in view of an investigation of the optical propagation properties of the $\varphi_{n, E}^{(N)}$, s, the curves in the graphs can be regarded as the profiles of the elegant HL and HG beams along the transverse coordinate on the initial plane, usually taken at $z=0, z$ denoting the propagation variable.

As remarked earlier, the $\varphi_{n, S}^{(N)}$ 's form an orthonormal set. In contrast, the $\varphi_{n, E}^{(N)}$, s do not provide an orthonormal set of beams, although they can be normalized (see the Appendix for details). An orthonormal set of EHL beams, however, could be constructed by means of the well known Gram-Schmidt method, also applied in [18] to construct an orthonormal set of super-Lorentzian beams.

Besides, the EHG beams $\varphi_{n, E}^{(\infty)}(\xi)$ form a biorthogonal set of functions with the pure Hermite polynomials $H_{n}(\xi)$. A similar biorthogonality relation for the $\varphi_{n, E}^{(N)}$, s with respect the $H_{n}^{(N)}$ 's does not seem to hold. In fact, even though the sets $\left\{\varphi_{n, E}^{(N)}\right\}_{n}$ and $\left\{H_{n}^{(N)}\right\}_{n}$ are eigenfunctions of adjoint operators, just as the $\varphi_{n, E}^{(\infty)}$ 's and the $H_{n}$ 's, the inner product $\left\langle H_{m}^{(N)} \mid \varphi_{n, E}^{(N)}\right\rangle$ does not vanish for any $m \neq n$. It is easily seen that it vanishes for any $m$ and $n$ such that $m+n$ be odd, having in contrast a rather various behavior when $m+n$ is even. In particular, for a given $n$, it vanishes for $m<n$, takes a finite value for $n \leq m<2 N+$ $n$ (specifically equal to $\left.\mathcal{N}_{n, N}^{(E)} \frac{m ! \Gamma\left(N+\frac{1}{2}\right)}{N^{\frac{n+m-1}{2}}} \frac{\Gamma(2 N+m)}{\Gamma(N) \Gamma(2 N)} \sum_{j=0}^{\left[\frac{m-n}{2}\right]} \frac{(-)^{j} \Gamma\left(N-\frac{m-n}{2}+j-\frac{1}{2}\right)}{4^{j} j !(m-n-2 j) !} \frac{\Gamma\left(\frac{m-n}{2}-j+\frac{1}{2}\right)}{\Gamma\left(N+j+\frac{1}{2}\right)}\right)$, whilst diverges for larger values of $m$.

\section{Hermite-Lorentzian beams: paraxial propagation properties}

In this section, we will investigate the paraxial propagation properties of the EHL beams $\varphi_{n}^{(N)}$, as given in $(2.15)^{(2)}$.

Various methods (evaluation of the Fresnel integral, combination of Fourier and antiFourier transforms with frequency chirping) can be pursued in order to work out the propagated form, i.e. at $z>0$, in the paraxial approximation, of a given (i.e. at $z=0$ ) optical field.

Here, we will resort to the Fourier transform/frequency chirping/inverse Fourier transform approach. Comments on the appropriateness of the paraxial approximation to account for the overall propagation properties of the $\varphi_{n}^{(N)}$ 's will be given below.

Before going on, let us briefly consider the operatorial expression for the paraxial propagation, from which of course the aforementioned methods can directly or indirectly be inferred; namely the expression

$$
\varphi_{n}^{(N)}(\xi, \zeta)=e^{i \frac{\zeta}{4} \frac{\partial^{2}}{\partial \xi^{2}}} \varphi_{n}^{(N)}(\xi)
$$

It follows from the $1 \mathrm{D}$ paraxial wave equation, which we take in the form $\left[4 i \frac{\partial}{\partial \zeta}+\frac{\partial^{2}}{\partial \xi^{2}}\right] u(\xi, \zeta)=$ 0 , in correspondence with the initial condition $u(\xi, 0)=\varphi_{n}^{(N)}(\xi)$. The variables $\xi, \zeta$ denote the transverse and longitudinal coordinates $x, z$ scaled respectively to the characteristic width $w_{0}$ and the characterisitic length $z_{R}=\frac{k w_{0}^{2}}{2}$ associated with a Gaussian beam, i.e. $\xi \equiv \frac{x}{w_{0}}$ and $\zeta \equiv \frac{z}{z_{R}}$. This will be the meaning of the variables $\xi$ and $\zeta$ in the following.

\footnotetext{
${ }^{2}$ Since in the following we will consider throughout the elegant HL functions $\varphi_{n, E}^{(N)}$, for ease of notation we will omit the subscript E.
} 
Therefore, when talking of "limiting" or "corresponding" EHG beams in the sense of the aforementioned limit relation, EHG beams with characteristic width $w_{0}$ will be intended.

Since the $\varphi_{n}^{(N)}$ 's are nothing but the $n$-th derivatives of the Lorentz beams of pertinent parameter $N: \varphi_{n}^{(N)}(\xi) \propto(-)^{n} \frac{d^{n}}{d \xi^{n}}\left(1+\frac{\xi^{2}}{N}\right)^{-N}$, we see that

$$
\varphi_{n}^{(N)}(\xi, \zeta)=e^{i \frac{\zeta}{4} \frac{\partial^{2}}{\partial \xi^{2}}} \mathcal{N}_{n, N}(-)^{n} \frac{d^{n}}{d \xi^{n}}\left(1+\frac{\xi^{2}}{N}\right)^{-N}=\frac{\mathcal{N}_{n, N}}{\mathcal{N}_{0, N}}\left(-\frac{\partial}{\partial \xi}\right)^{n} e^{i \frac{\zeta}{4} \frac{\partial^{2}}{\partial \xi^{2}}} \varphi_{0}^{(N)}(\xi)
$$

One is therefore led to investigate the propagation properties of the fundamental HL beams $\varphi_{0}^{(N)}(\xi)$ of relevant parameter $N$ according to

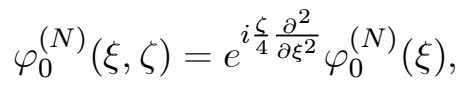

in the same way as, in the case of the EHG beams, one is primarily concerned with the propagation of the fundamental Gaussian-beam $e^{-\xi^{2}}$.

Expressing the operator in (3.3) in the form of the inherent power series and exploiting the Rodrigues formula (2.13), we obtain the propagated beam

$$
\varphi_{0}^{(N)}(\xi, \zeta)=\mathcal{N}_{0, N} \sum_{n=0}^{\infty} \frac{1}{n !}\left(i \frac{\zeta}{4}\right)^{n} \frac{\partial^{2 n}}{\partial \xi^{2 n}}\left(1+\frac{\xi^{2}}{N}\right)^{-N}=\mathcal{N}_{0, N} \sum_{n=0}^{\infty} \frac{1}{n !}\left(i \frac{\zeta}{4}\right)^{n} u_{2 n}^{(N)}
$$

as an infinite series involving the unnormalized HL beams of even order $u_{2 n}^{(N)} \equiv \frac{1}{\mathcal{N}_{n, N}^{(E)}} \varphi_{n, E}^{(N)}$, as expected in accord with the symmetry properties of the free space.

Expression (3.4) signals that the propagation of the fundamental HL beams $\left(1+\frac{\xi^{2}}{N}\right)^{-N}$ naturally amounts to the production of all the relevant even-order modes.

Unfortunately, the evaluation to a closed form of the series in (3.4), which is a sort of generating function of the RHPs involving only the even-order polynomials, does not appear to be easily obtainable for finite values of $N$ through a direct calculation - apart from the case $N=1$, as we will briefly discuss in the next section.

In the Gaussian limit $N \rightarrow \infty$ the series in (3.4) results in $e^{-\xi^{2}} \sum_{n=0}^{\infty} \frac{1}{n !}\left(i \frac{\zeta}{4}\right)^{n} H_{2 n}(\xi)$, which, on account of the expression for the generating function of the even-order Hermite polynomials, immediately yields the well known propagation rule for the Gaussian beam:

$$
\varphi_{0}^{(\infty)}(\xi, \zeta)=\left(\frac{2}{\pi}\right)^{\frac{1}{4}} \frac{1}{\sqrt{1+i \zeta}} e^{-\frac{\xi^{2}}{1+i \zeta}}
$$

\subsection{Hermite-Lorentzian beams of multiplicity parameter $N=1$}

Let us firstly consider the normalized Lorentz beam

$$
\varphi_{0}^{(1)}(\xi)=\sqrt{\frac{2}{\pi}} \frac{1}{1+\xi^{2}}
$$

and the associated higher-order modes $\varphi_{n}^{(1)}$.

As noted earlier, the propagation properties of the beam (3.6) have been analyzed in detail in [1], where the relevant propagated form has been deduced (see Eq. (15) therein) through the Fourier transform/frequency chirping/anti-Fourier transform method. We will reconsider here such a propagated form and use it to infer those relevant to the higher-order modes in accord with (3.2). 
So, going through the aforementioned procedure in relation with the wave form in (3.6), for the range of $\zeta$-values of our interest (i.e. $\zeta>0$ ) we obtain

$$
\varphi_{0}^{(1)}(\xi, \zeta)=\frac{1}{\sqrt{\pi i \zeta}}\left\{e^{\frac{(1+i \xi)^{2}}{2 i \zeta}} D_{-1}\left(\sqrt{2} \frac{1+i \xi}{\sqrt{i \zeta}}\right)+e^{\frac{(1-i \xi)^{2}}{2 i \zeta}} D_{-1}\left(\sqrt{2} \frac{1-i \xi}{\sqrt{i \zeta}}\right)\right\},
$$

where $D_{\nu}$ denotes the Weber-Hermite (or parabolic cylinder) function. Evidently, the above expression coincides with that worked out in [1] in virtue of that the Weber-Hermite function of negative integer order relates to the derivatives of the erfc function as [15]

$$
D_{-n-1}(z)=\frac{(-)^{n}}{n !} \sqrt{\frac{\pi}{2}} e^{-\frac{z^{2}}{4}} \frac{d^{n}}{d z^{n}}\left[e^{\frac{z^{2}}{2}} \operatorname{erfc}\left(\frac{z}{\sqrt{2}}\right)\right] .
$$

The notation in terms of the parabolic cylinder function seems to be more appealing in view of the results presented in $[4,5]$, where a certain class of general solutions of the 1D paraxial wave equation in free space has been proved to have as its central part just the Weber-Hermite function, modulated by a complex quadratic exponential. In fact, as mentioned earlier, in [4] the link of the Lorentz beam (3.6) with such solutions has been clarified. We will comment on this in Sect. 3.3.

The asymptotic expansion $D_{\nu}(z) \approx e^{-\frac{z^{2}}{4}} z^{\nu}$ [15], valid for large values of $|z|,|z| \gg|\nu|$ and $|\arg (z)|<\frac{3 \pi}{4}$, allows us to recover the Lorentz wave-form (3.6) in the limit $\zeta=0$.

As earlier remarked, the expression (3.7) may also be obtained from the evaluation of the series in (3.4) relative to the case $N=1$, namely

$$
S^{(1)}(\xi, \zeta)=\frac{1}{1+\xi^{2}} \sum_{n=0}^{\infty} \frac{1}{n !}\left(i \frac{\zeta}{4}\right)^{n}\left(\frac{1}{1+\xi^{2}}\right)^{-2 n} H_{2 n}^{(1)}(\xi) .
$$

We may go through the identities

$$
H_{2 n}^{(1)}(\xi)=(2 n) !\left(1+\xi^{2}\right)^{n} C_{2 n}^{1}\left(\frac{\xi}{\sqrt{1+\xi^{2}}}\right)=\frac{(-)}{2}^{n}(2 n) !\left\{(1+i \xi)^{2 n+1}+(1-i \xi)^{2 n+1}\right\},
$$

the last resulting from that $C_{n}^{1}(x)=U_{n}(x)=\frac{1}{2 i} \frac{\left(x+i \sqrt{1-x^{2}}\right)^{n+1}-\left(x-i \sqrt{1-x^{2}}\right)^{n+1}}{\sqrt{1-x^{2}}}$, where $U_{n}(x)$ is the Chebyshev polynomial of the second type [15]. Accordingly, it turns out that

$$
S^{(1)}(\xi, \zeta)=\frac{1}{2(1+i \xi)}{ }_{2} F_{0}\left(\frac{1}{2}, 1 ;-; \frac{-i \zeta}{(1+i \xi)^{2}}\right)+\frac{1}{2(1-i \xi)}{ }_{2} F_{0}\left(\frac{1}{2}, 1 ;-; \frac{-i \zeta}{(1-i \xi)^{2}}\right),
$$

which, by suitably recasting the hypergeometric series ${ }_{2} F_{0}\left(\frac{1}{2}, 1 ;-; z\right)$ on account of that $\left(\frac{1}{2}\right)_{n}=\frac{1}{2^{2 n} n !} \int_{0}^{\infty} t^{2 n} e^{-t} d t$, leads to the expression (3.7) for $\varphi_{0}^{(1)}(\xi, \zeta)$.

The $\xi$ - $\zeta$ behavior of the Lorentz-beam amplitude $\left|\varphi_{0}^{(1)}(\xi, \zeta)\right|$ is displayed in the 3D plot of part (a) of Fig. 3.1, part (b) of which allows us to grasp the difference of behavior of $\left|\varphi_{0}^{(1)}(\xi, \zeta)\right|$ with respect to the corresponding Gaussian beam amplitude by displaying in the same frame the surface plots of both.

As to the higher-order modes $\varphi_{n}^{(1)}$, according to (3.2) we simply have

$$
\varphi_{n}^{(1)}(\xi, \zeta)=\frac{2^{n}}{\sqrt{\Gamma(2 n+1)}}\left(-\frac{\partial}{\partial \xi}\right)^{n} \varphi_{0}^{(1)}(\xi, \zeta),
$$




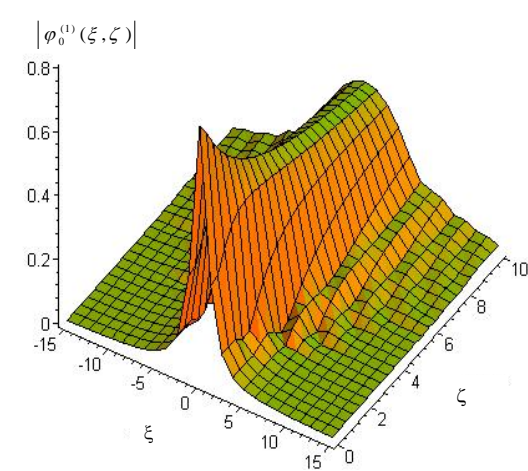

(a)

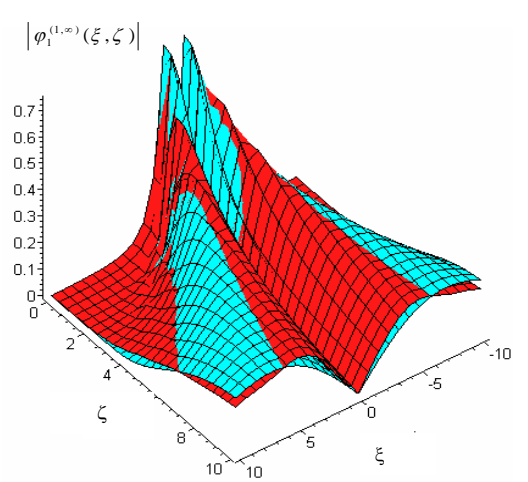

(c)

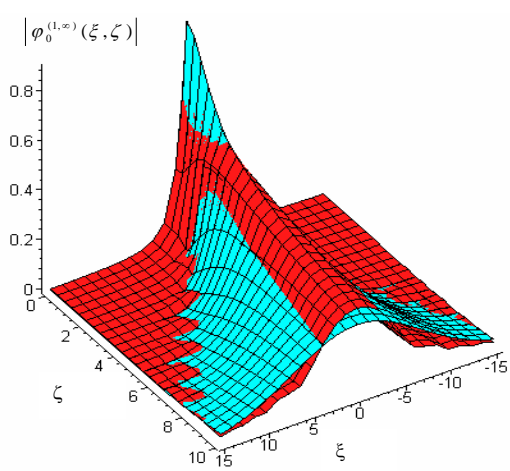

(b)

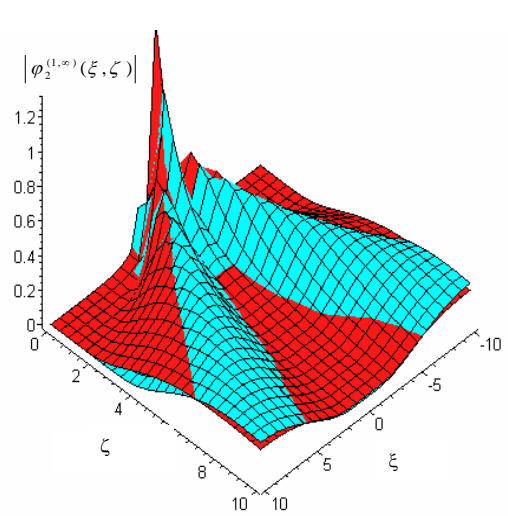

(d)

Figure 3.1: $\xi$ - $\zeta$ behavior of (a) the Lorentz-beam amplitude $\left|\varphi_{0}^{(1)}(\xi, \zeta)\right|$ and of the Lorentz and the corresponding EHG beam amplitudes $\left|\varphi_{n}^{(1)}(\xi, \zeta)\right|$ (dark color surface) and $\left|\varphi_{n}^{(\infty)}(\xi, \zeta)\right|$ (clear color surface) of order (b) $n=0$, (c) $n=1$ and (d) $n=2$.

which, by virtue of the property of the parabolic cylinder functions: $\frac{d^{m}}{d z^{m}}\left[e^{\frac{z^{2}}{4}} D_{\nu}(z)\right]=$ $(-)^{m}(-\nu)_{m} e^{\frac{z^{2}}{4}} D_{\nu-m}(z)$ [15] (or, equivalently of (3.8)), recasts in the parity preserving superposition of parabolic cylinder functions of suited negative order:

$\varphi_{n}^{(1)}(\xi, \zeta)=\sqrt{\frac{2^{n} n !}{\sqrt{\pi} \Gamma\left(n+\frac{1}{2}\right)}} i^{n}\left(\frac{1}{i \zeta}\right)^{\frac{n+1}{2}}\left\{e^{\frac{(1+i \xi)^{2}}{2 i \zeta}} D_{-n-1}\left(\sqrt{2} \frac{1+i \xi}{\sqrt{i \zeta}}\right)+(-)^{n} e^{\frac{(1-i \xi)^{2}}{2 i \zeta}} D_{-n-1}\left(\sqrt{2} \frac{1-i \xi}{\sqrt{i \zeta}}\right)\right\}$.

A certain insight into the behavior of the higher-order beam amplitudes can be gained from the pictures in Fig. 3.1.(c) and (d), where the surface plots of the beam amplitudes $\left|\varphi_{1,2}^{(1)}(\xi, \zeta)\right|$ are, for comparison purposes, shown alongside those of the limiting EHG beams. As is well known, the latter propagate according to

$$
\varphi_{n, E}^{(\infty)}(\xi, \zeta)=\frac{1}{\sqrt{2^{n-\frac{1}{2}} \Gamma\left(n+\frac{1}{2}\right)}}\left(\frac{1}{\mu(\zeta)}\right)^{\frac{n+1}{2}} e^{-\frac{\xi^{2}}{\mu(\zeta)}} H_{n}\left(\frac{\xi}{\sqrt{\mu(\zeta)}}\right), \quad \mu(\zeta) \equiv 1+i \zeta .
$$

Also, in Fig. 3.2 the $\xi-\zeta$ contourplots of $\left|\varphi_{0,1,2}^{(1)}(\xi, \zeta)\right|$ are shown again along with those of the corresponding EHG beams of the same orders. 


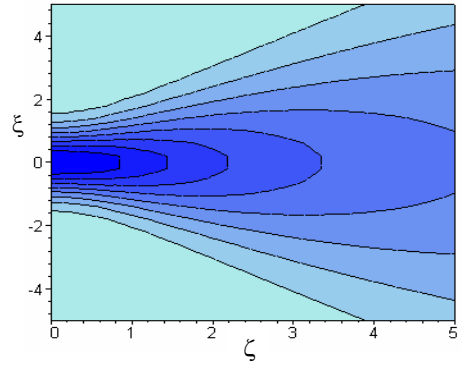

(a)

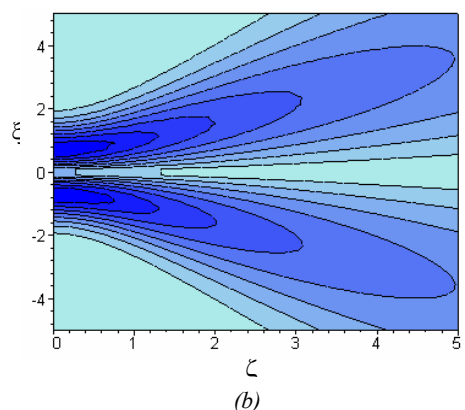

(b)

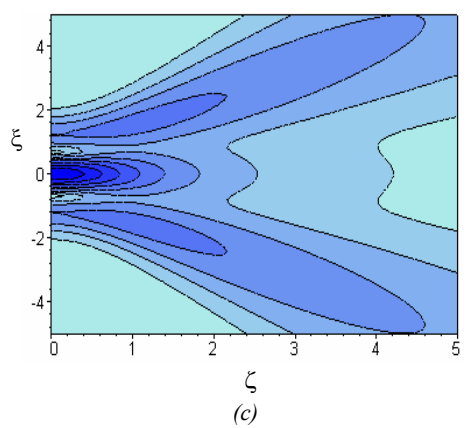

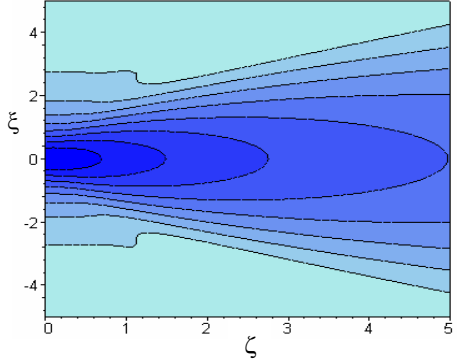

(d)
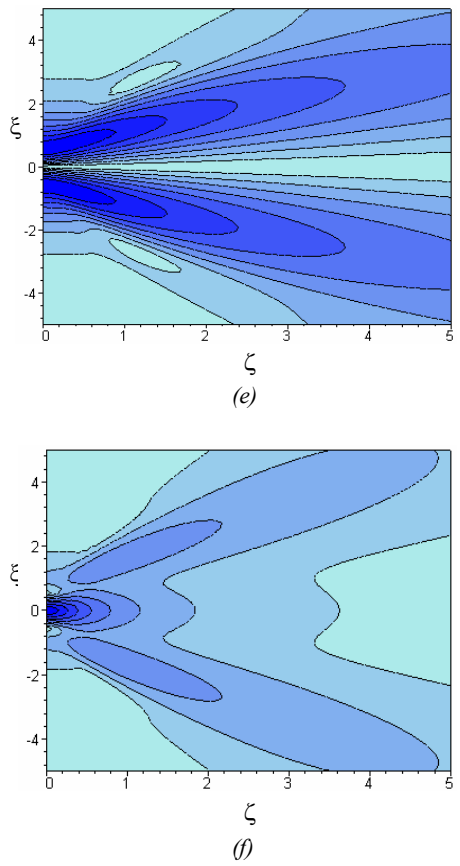

Figure 3.2: $\xi-\zeta$ contourplots of $(d),(e),(f)$ the EHL beam amplitudes respectively of order $n=0,1,2$ and of $(a),(b),(c)$ the corresponding EHG beam amplitudes of the same order.

We see that the spreading of the Lorentz waveform (3.6) is smoother than that of the limiting Gaussian beam. More precisely, using the asymptotic expansion of the parabolic cylinder function, we can approximate the function (3.7) as

$$
\varphi_{0}^{(1)}(\xi, \zeta)=\sqrt{\frac{2}{\pi}} \frac{1}{1+\xi^{2}}\left\{1-i \frac{\zeta}{2} \frac{1-3 \xi^{2}}{\left(1+\xi^{2}\right)^{2}}\right\}
$$

for $\zeta \leq 0.1$. The relevant spatial variance $\sigma_{0,1}^{2}(\zeta)$ evaluates therefore to

$$
\sigma_{0,1}^{2}(\zeta)=1+\frac{\zeta^{2}}{32}
$$

whilst for the corresponding Gaussian beam one has $\sigma_{0, \infty}^{2}(\zeta)=\frac{1}{4}\left(1+\zeta^{2}\right)$ for $\zeta \geq 0{ }^{(3)}$.

\footnotetext{
${ }^{3}$ A more detailed analysis of the spreading properties of the Lorentz and Gaussian beams, within and beyond the paraxial regime, can be performed in the context of the recently introduced paraxiality-degree related formalism $[19,20]$.
} 
A rather similar behavior is exhibited by the mode of order $n=1$, whilst it is evident that the intrinsic divergence of the modes increases rather rapidly for $n \geq 2$. This is due to the fact that the "width" of the $\varphi_{n}^{(1)}$ 's at $\zeta=0$ quickly decreases for $n \geq 2$, because of the factor $\left(1+\xi^{2}\right)^{-n-1}$ which modulates the polynomial $H_{n}^{(1)}$. Then, the relevant divergence increases, as a consequence of the optical uncertainty relation (see the Appendix for more quantitative considerations). Such a feature of the chosen set of the $\varphi_{n}^{(1)}(\xi)$ - and, more in general, of the $\varphi_{n}^{(N)}(\xi)$ - may generate legitimate doubts about the appropriateness of the paraxial approximation to account for their overall behaviour at $\zeta>0$. Perhaps, more convenient sets of $\varphi_{n}^{(N)}$ 's might be envisaged as suggested in the concluding notes.
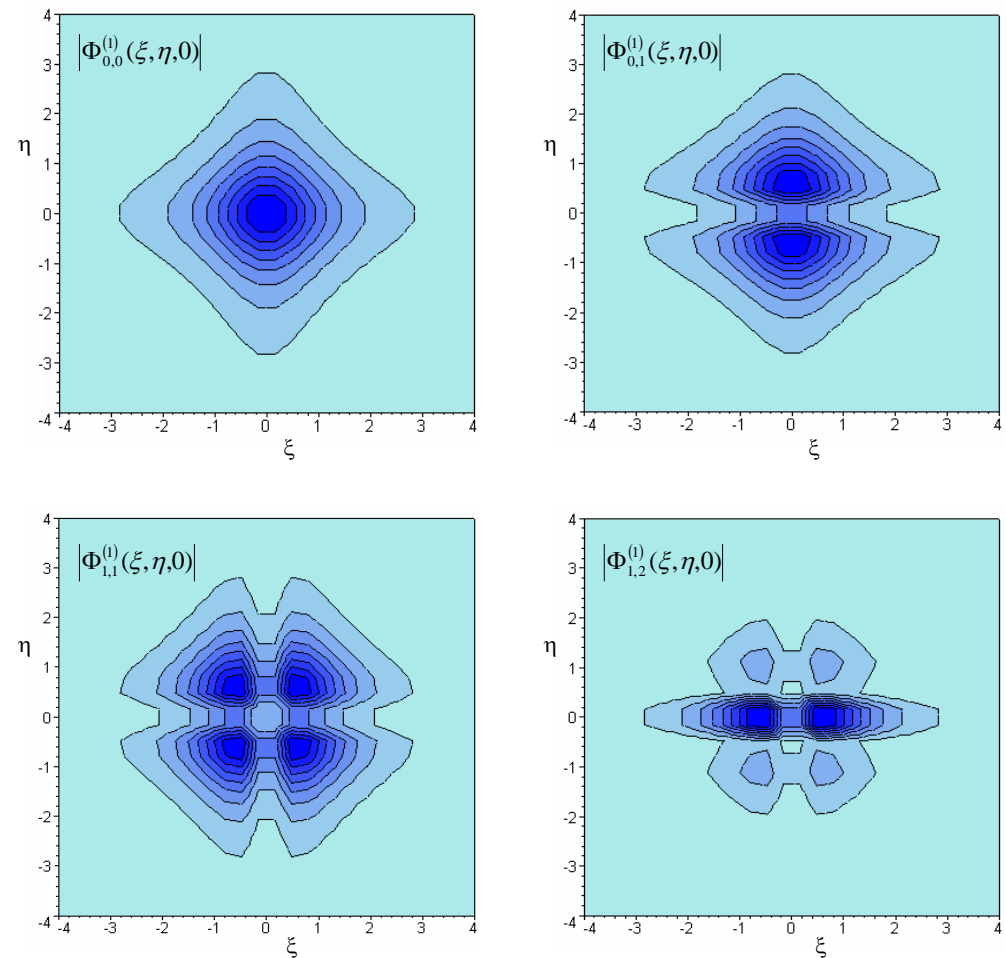

Figure 3.3: Amplitude distributions of the $2 D$ EHL beams $\Phi_{n, m}^{(1)}(\xi, \eta, \zeta)$ at $\zeta=0$ for some index pairs $(n, m)$.

Finally, a view of the possible amplitude and phase behavior of the $2 \mathrm{D} N=1 \mathrm{EHL}$ beams, obtainable, of course, as products of the relevant 1D beams:

$$
\Phi_{n, m}^{(1)}(\xi, \eta, \zeta)=\varphi_{n}^{(1)}(\xi, \zeta) \varphi_{m}^{(1)}(\eta, \zeta)
$$

is offered by the contourplots in Figs. 3.3-3.5. Figure 3.3 shows the amplitude distributions at $\zeta=0$ of the $\Phi_{n, m}^{(1)}$ 's for some values of the indices $n$ and $m$, the relevant amplitude and phase distributions at $\zeta=1$ are then displayed in the plots of Figs. 3.4 and 3.5.

The considered modes have specific symmetry properties, which for some of them are preserved by propagation. So, we may see that the fundamental mode $\Phi_{0,0}^{(1)}$ has an expected rectangular symmetry, which is preserved by propagation as displayed by the phase distribution shown in Fig. 3.4. In turn, the other modes display an initial symmetry for 

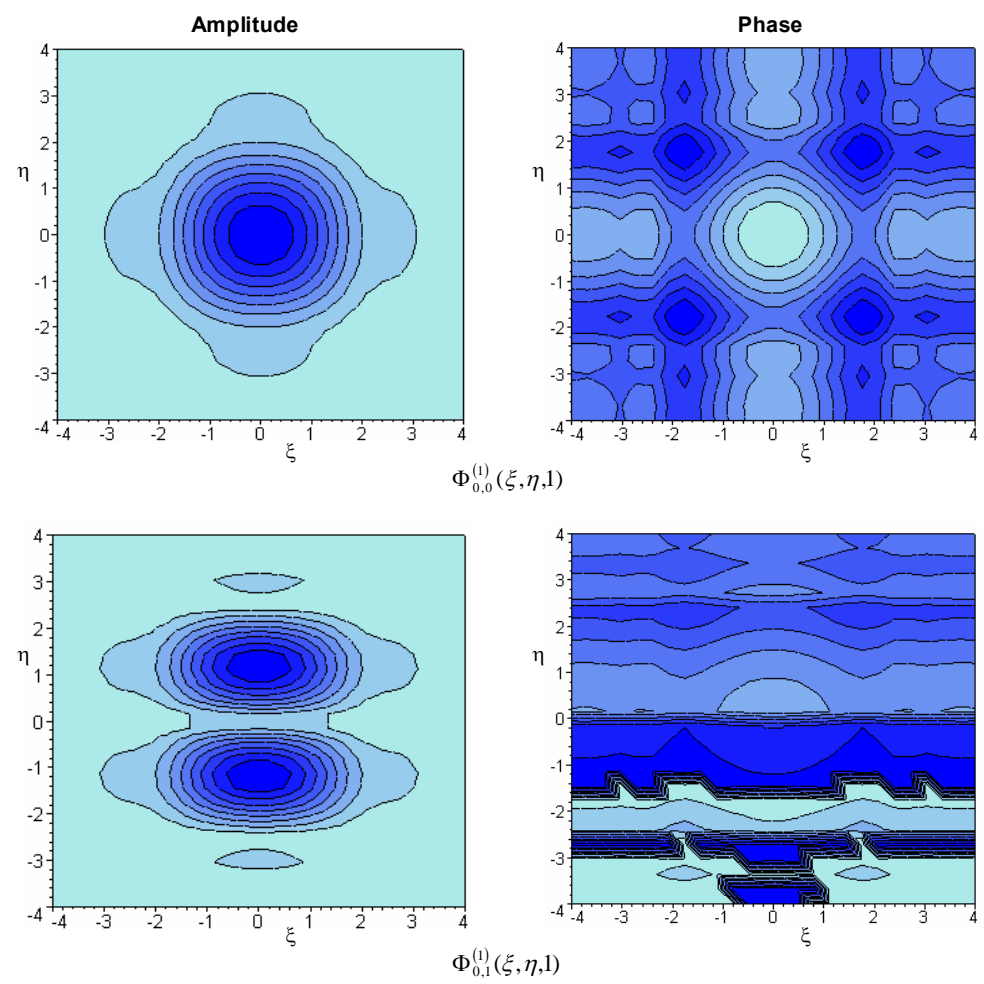

Figure 3.4: Amplitude and phase distributions of the $2 D$ EHL beams $\Phi_{n, m}^{(1)}(\xi, \eta, \zeta)$ at $\zeta=1$ for the index pairs $(0,0)$ and $(0,1)$.

inversion with respect to the origin $\xi=\eta=0$; which symmetry is preserved by propagation in the case of the mode $\Phi_{1,1}^{(1)}$, as confirmed by the relevant phase distribution in Fig. 3.5 , but not in that of the modes $\Phi_{0,1}^{(1)}$ and $\Phi_{1,2}^{(1)}$. As to the latter, we may signalize an interesting behavior: the amplitude distribution of this mode at $\zeta=0$ seems in fact to be mainly dominated by the 1D mode $\varphi_{1}^{(1)}(\xi)$, whilst, as we see from Fig. 3.5, the other 1D component mode, i.e. $\varphi_{2}^{(1)}(\xi)$, more strongly emerges to shape the $2 \mathrm{D}$ mode at $\zeta=1$.

\subsection{Hermite-Lorentzian beams of multiplicity parameter $N>1$}

Let us consider now the Lorentz beam of multiplicity parameter $N>1$ :

$$
\varphi_{0}^{(N)}(\xi)=\mathcal{N}_{0, N} \frac{1}{\left(1+\frac{\xi^{2}}{N}\right)^{N}}
$$

with $\mathcal{N}_{0, N}=\sqrt{\frac{\Gamma(2 N)}{\sqrt{\pi N} \Gamma\left(2 N-\frac{1}{2}\right)}}$.

Going again through the Fourier transform/frequency chirping/inverse Fourier transform procedure in relation with the wave form (3.13), we obtain, as one may expect in accord with the multiplicity $N$ of the pairs of poles $\xi_{0}= \pm i \sqrt{N}$ of (3.13) in the complex plane, a sum of $N$ terms, each reproducing the functional structure of (3.7) with parabolic cylinder functions of different orders and suitably different argument (see also Eq. (39) in 

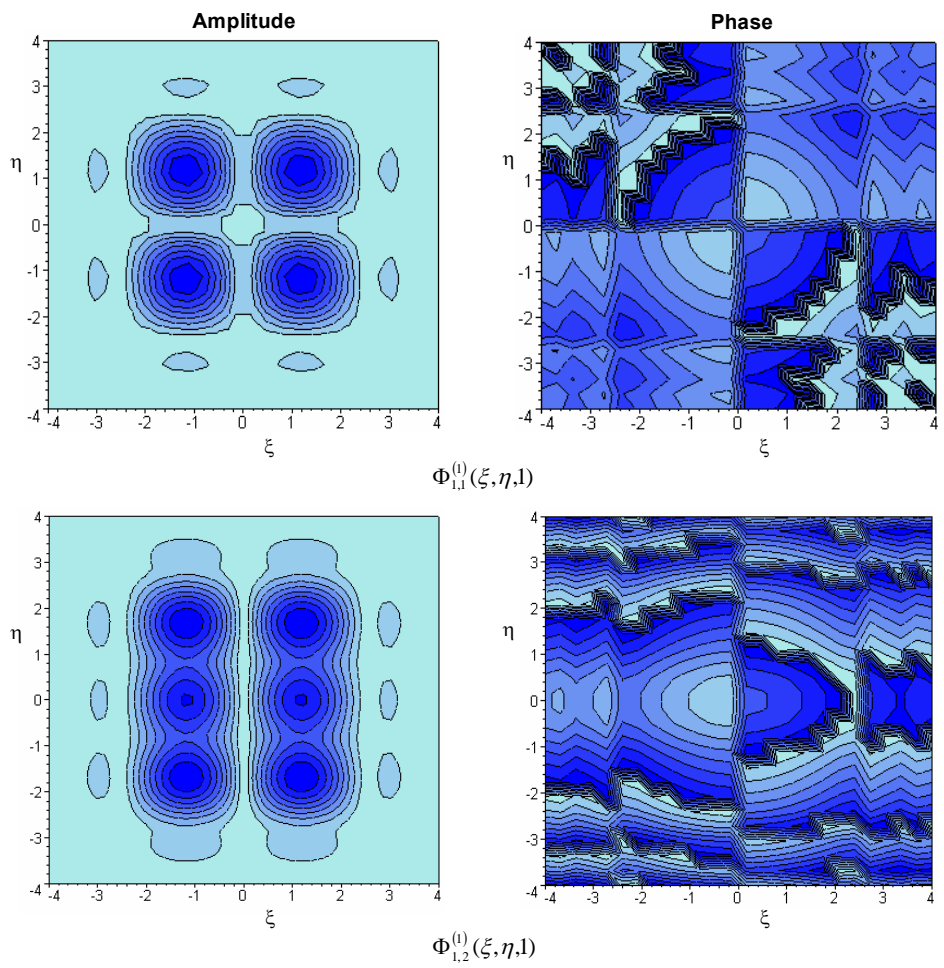

Figure 3.5: Amplitude and phase distributions of the $2 D$ EHL beams $\Phi_{n, m}^{(1)}(\xi, \eta, \zeta)$ at $\zeta=1$ for the index pairs $(1,1)$ and $(1,2)$.

[18]). Explicitly, we have ${ }^{(4)}$

$$
\begin{gathered}
\varphi_{0}^{(N)}(\xi, \zeta)=\mathcal{N}_{0, N} \sum_{j=0}^{N-1} \frac{(N)_{j}}{4^{j} j !}\left(\frac{N}{2 i \zeta}\right)^{\frac{N-j}{2}} \\
\times\left\{e^{\frac{(\sqrt{N}+i \xi)^{2}}{2 i \zeta}} D_{-N+j}\left(\sqrt{2} \frac{\sqrt{N}+i \xi}{\sqrt{i \zeta}}\right)+e^{\frac{(\sqrt{N}-i \xi)^{2}}{2 i \zeta}} D_{-N+j}\left(\sqrt{2} \frac{\sqrt{N}-i \xi}{\sqrt{i \zeta}}\right)\right\} .
\end{gathered}
$$

As to the higher order modes, applying as before Eq. (3.2), we end up with the sum of $N$ terms each being the symmetric or antisymmetric superposition - according to the modeorder evenness - of the basic "block" $e^{\frac{z^{2}}{2}} D_{-N+j-n}(\sqrt{2} z)$, with arguments respectively

${ }^{4}$ For completeness'sake we note that the Fourier integral of the multi-Lorentzian (3.13) writes as

$$
\begin{aligned}
{\left[\widehat{\mathcal{F}} \varphi_{0}^{(N)}\right](\kappa) \equiv } & \frac{1}{\sqrt{2 \pi}} \int_{-\infty}^{+\infty} e^{-i \kappa \xi} \varphi_{0}^{(N)}(\xi) d \xi=\sqrt{\frac{N \Gamma\left(N+\frac{1}{2}\right)}{\Gamma(N) \Gamma\left(2 N-\frac{1}{2}\right)}}|\kappa|^{N-1} W_{0, N-\frac{1}{2}}(2 \sqrt{N}|\kappa|) \\
& =\frac{(-)^{N-1}}{2^{N-1}} \sqrt{\frac{\sqrt{N} \Gamma(N) \Gamma\left(N+\frac{1}{2}\right)}{\Gamma\left(2 N-\frac{1}{2}\right)}} e^{-\sqrt{N}|\kappa|} L_{N-1}^{(1-2 N)}(2 \sqrt{N}|\kappa|)
\end{aligned}
$$

where $W_{v, \mu}$ denotes Whittaker's second function and $L_{n}^{(\alpha)}$ the generalized Laguerre polynomial [21]. We also recall that $W_{0, \mu}(z)=\sqrt{\frac{z}{\pi}} K_{\mu}\left(\frac{z}{2}\right), K_{\mu}$ being the modified Bessel function of second type. In particular, the Fourier transform of $\varphi_{0}^{(1)}(\xi):\left[\widehat{\mathcal{F}} \varphi_{0}^{(1)}\right](\kappa)=e^{-|\kappa|}$ shapes as a rather thin cusp. Such a cusp disappears already for $N=2$, the Fourier transform of $\varphi_{0}^{(2)}(\xi)$ dispalying in fact a smoother shape. 
$z=\frac{\sqrt{N}+i \xi}{\sqrt{i \zeta}}$ and $z=\frac{\sqrt{N}-i \xi}{\sqrt{i \zeta}}$

$$
\begin{gathered}
\varphi_{n}^{(N)}(\xi, \zeta)=\mathcal{N}_{n, N}\left(\frac{2 i}{\sqrt{N}}\right)^{n} \sum_{j=0}^{N-1} \frac{(N)_{j}(N-j)_{n}}{4^{j} j !}\left(\frac{N}{2 i \zeta}\right)^{\frac{N-j+n}{2}} \\
\times\left\{e^{\frac{(\sqrt{N}+i \xi)^{2}}{2 i \zeta}} D_{-N+j-n}\left(\sqrt{2} \frac{\sqrt{N}+i \xi}{\sqrt{i \zeta}}\right)+(-)^{n} e^{\frac{(\sqrt{N}-i \xi)^{2}}{2 i \zeta}} D_{-N+j-n}\left(\sqrt{2} \frac{\sqrt{N}-i \xi}{\sqrt{i \zeta}}\right)\right\},
\end{gathered}
$$

with $\mathcal{N}_{n, N}$ given before.

As a completion of Fig. 3.2, Fig. 3.6 shows the $\xi-\zeta$ contourplots of the beam amplitudes $\left|\varphi_{0,1,2}^{(N)}(\xi, \zeta)\right|$ for $N=2$ and $N=5$. We see that the behavior for $N=5$ more nearly resembles that of the corresponding EHGBs, as displayed in the contourplots of Fig. 3.2.

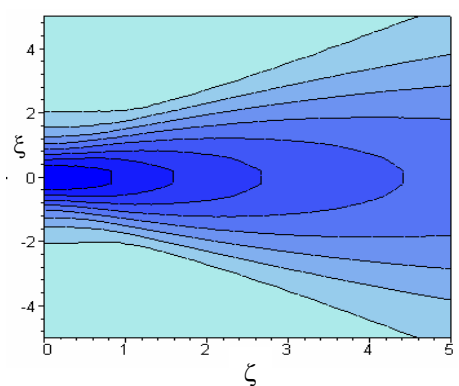

(a)

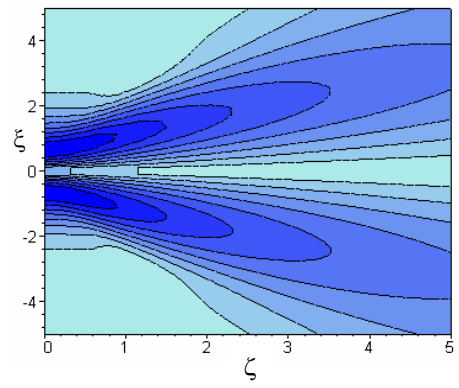

(c)

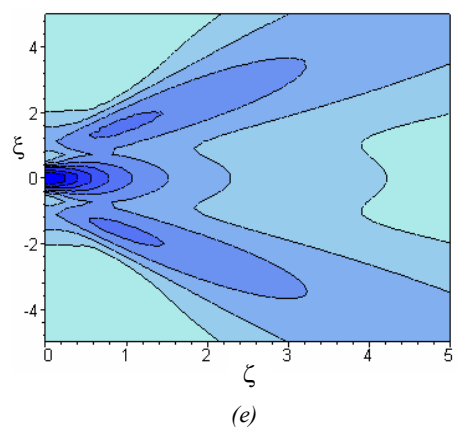

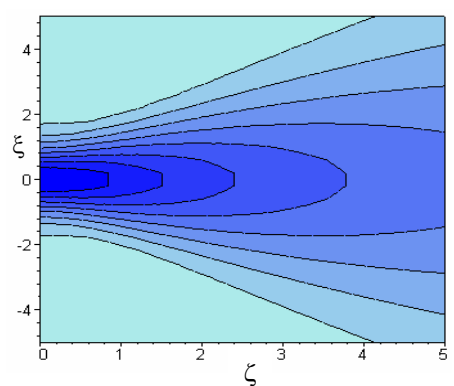

(b)

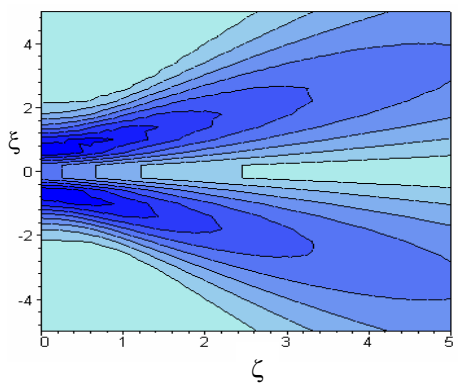

(d)

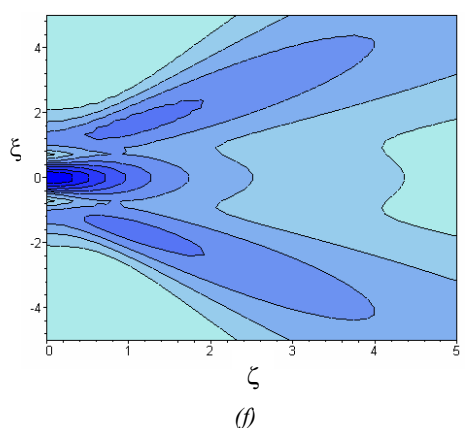

Figure 3.6: $\xi$ - $\zeta$ contourplots of the EHL beam amplitudes $\left|\varphi_{n}^{(N)}(\xi, \zeta)\right|$ with (a) $n=0, N=2$; (b) $n=0, N=5$; (c) $n=1, N=2$; (d) $n=1, N=5$; (e) $n=2, N=2$ and (f) $n=2, N=5$. 


\subsection{Hermite-Lorentzian and Weber-Hermite beams}

As mentioned earlier, a recent re-analysis of the 1D paraxial wave equation in free space yielded a certain class of general separable-variable based solutions of it $[4,5]$.

These solutions have a structure, basically comprising an in general complex quadratic exponential times a modulating Weber-Hermite function $D_{\nu}$ of suitable argument, such that even and odd waveforms with respect to the spatial coordinate can be taken. Also, it depends on three free parameters, denoted as $\left(\lambda, \zeta_{0}, \bar{\zeta}_{0}\right)$ in [5], two of which, $\lambda$ and $\bar{\zeta}_{0}$, within the formalism of that paper, have been related to the eigenvalues and relative eigenfunctions of a given symmetry operator of the equation and the other, $\zeta_{0}$, to the propagation features. More precisely, $\lambda$ comes to represent the eigenvalues of the chosen symmetry operator of the wave equation, whose eigenfunctions, further specified by the other parameter $\bar{\zeta}_{0}$, act as a sort of "source" functions, which once propagated from $\zeta_{0}$ generate the solution at hand. The final result, as to the transverse dependence, turns out to be the parabolic-cylinder function $D_{i \lambda-\frac{1}{2}}$, sandwiched between two complex quadratic exponentials whose parameters $Q(\zeta)=\zeta-\zeta_{0}$ and $\bar{Q}(\zeta)=\zeta-\bar{\zeta}_{0}$ are seen to be "formed" by the interplay of the propagation and "source" function features, and suitably combine to shape the scaling factor of the spatial coordinate in the argument of $D_{i \lambda-\frac{1}{2}}$.

Properly specifying the three parameters $\lambda, \zeta_{0}$ and $\bar{\zeta}_{0}$, yields all the modes discussed in the literature. In fact, in [4] a complete table of correspondences is displayed in terms of the aforementioned even and odd modes. In particular, for the case of our interest, setting the inherent parameters so that $Q(\zeta) / \zeta_{0}=$ const., $\bar{Q}(\zeta)=\zeta$ and $\lambda=i\left(n+\frac{1}{2}\right), n$ non-negative integer, one gets the building "blocks" of the EHL modes for $N=1$, i.e.

$$
W_{n, N}^{( \pm)}(\xi, \zeta)=\left(\frac{1}{i \zeta}\right)^{\frac{n+1}{2}} D_{-n-1}\left( \pm e^{i \frac{\pi}{4}} \sqrt{\frac{2}{\zeta}} \xi\right) e^{\frac{\xi^{2}}{2 i \zeta}}, \quad n=0,1,2 \ldots
$$

It becomes therefore clear that the EHL beams $\varphi_{n}^{(1)}(\xi, \zeta)$ are obtained by combining such building blocks through an even or odd superposition according to the evenness of the beam order $n$, after affecting them respectively by the shifts $-i$ and $+i^{(5)}$; namely,

$$
\varphi_{n}^{(1)}(\xi, \zeta) \propto W_{n, N}^{(+)}(\xi-i, \zeta)+(-)^{n} W_{n, N}^{(-)}(\xi+i, \zeta) .
$$

The same basic blocks (3.16) serve to form the EHL modes of parameter $N>1$. In this case, a definite sum of symmetric superpositions of the $W_{p, N}^{( \pm)}$, s from $p=0$ to $p=N-1$, further shifted by $\mp i \sqrt{N}$, is needed to form the fundamental beam $\varphi_{0}^{(N)}(\xi, \zeta)$, whereas an analogous sum of $n$-evenness dependent symmetric or antisymmetric superpositions of the similarly shifted $W_{p+n, N}^{( \pm)}$'s is seen to shape the higher-order beams $\varphi_{n}^{(N)}(\xi, \zeta)$.

\section{Conclusions}

We have evidenced the link between the relativistic Hermite polynomials (which are basically Gegenbauer polynomials), introduced within a purely quantum mechanical context [6], and the Lorentz beams, which, suggested by experimental observations as possible optical wave-fields, have been analyzed in detail in the literature $[1,18,4]$. Such a link naturally arises from the Rodrigues formula pertaining to the RHPs, which, accordingly,

\footnotetext{
${ }^{5}$ Note that, since the translation operator $e^{\delta \frac{\partial}{\partial \xi}}$ commutes with the propagation operator $e^{i \frac{\zeta}{4} \frac{\partial^{2}}{\partial \xi^{2}}}$, such shifts could also be acted on the "source" functions themselves.
} 
suggests the definition of what we have referred to as the (elegant) Hermite-(or, Gegenbauer-)Lorentzian beams, as given in (2.15). Their optical implementation seems to be feasible due to the well known derivative rule: $\widehat{\mathcal{F}} \frac{d^{n}}{d \xi^{n}}=(i \xi)^{n} \widehat{\mathcal{F}}$, obeyed by the Fourier transform, which amounts indeed to the relation $\frac{d^{n}}{d \xi^{n}}=\widehat{\mathcal{F}}^{-1}(i \xi)^{n} \widehat{\mathcal{F}}$, each operator in it having its own definite optical counterpart.

Once considered from the viewpoint of the optical propagation, the introduced HL beams reveal interesting behavior, also characterized by the further parameter $N$, which in fact signals the difference between the HL and the HG beams, the latter being regained in the "non-relativistic" limit $N \rightarrow \infty$. In addition, we have seen that the paraxial propagation of the HLBs of multiplicity parameter $N$ is describable as the superposition of $N$ symmetric or antisymmetric combinations of the Weber-Hermite beams $[4,5]$ of suited parameters $\left(\lambda, \zeta_{0}, \bar{\zeta}_{0}\right)$ and further shifted spatially by $\mp i \sqrt{N}$.

In the analysis here developed the functions $\varphi_{n, E}^{(N)}$ have been considered for "parallel" sets corresponding to given values of $N$. Of course, "transversal" sets may be considered as well, i.e. for a given $n$ and varying $N$. Thus, for instance, one might consider the functions $\varphi_{n, E}^{(N-n)}(\xi)$ for $N>n$ - already mentioned in Sec. 2 in connection with the wavelet transform issue approached in [10]. They will not suffer of the point source-like behavior for $n \rightarrow \infty$ as the $\varphi_{n, E}^{(N)}$ 's, that could be of some advantage in specific applications.

Let us note that a radial version of the $\varphi_{n, E}^{(N)}$, s, appropriate to a rotational symmetry, might be suggested by resorting to the definition of the relativistic Laguerre polynomials $[11]$,

$$
L_{n}^{(\alpha, N)}(\rho)=\sum_{j=0}^{n} \frac{1}{j !}\left(\begin{array}{c}
n+\alpha \\
n-m
\end{array}\right)\left(-n-N+\frac{1}{2}\right)_{j}\left(\frac{\rho}{N}\right)^{j}, \quad \alpha>-1, \quad 0 \leq \rho<\infty,
$$

for which the Rodrigues representation writes

$$
L_{n}^{(\alpha, N)}(\rho)=\frac{1}{n !}\left(1+\frac{\rho}{N}\right)^{N+2 n+\alpha+\frac{1}{2}} \rho^{-\alpha}\left(\frac{d}{d \rho}\right)^{n}\left\{\rho^{n+\alpha}\left(1+\frac{\rho}{N}\right)^{-N-n-\alpha-\frac{1}{2}}\right\} .
$$

As already noted, the above have been recognized just as Jacobi polynomials, being indeed $L_{n}^{(\alpha, N)}(\rho)=P_{n}^{\left(\alpha, \alpha-2 n-N-\frac{1}{2}\right)}\left(2 \frac{\rho}{N}+1\right)[9]$.

One may suggest introducing what can be referred to as (elegant) Laguerre-(or, Jacobi)Lorentzian beams of radial and azimuthal orders $n$ and $l$ respectively, according to

$$
\phi_{n, l, E}^{(N)}(\rho, \vartheta) \propto e^{i l \vartheta} \rho^{l} L_{n}^{(l, N)}\left(\rho^{2}\right)\left(1+\frac{\rho^{2}}{N}\right)^{-N-2 n-l-\frac{1}{2}},
$$

where $\rho$ and $\vartheta$ denote polar coordinates: $\rho^{2}=\xi^{2}+\eta^{2}$ and $\vartheta=\arctan \left(\frac{\eta}{\xi}\right)$. In the Gaussian limit $N \rightarrow \infty$ the above conforms to the well-known expression of the elegant LaguerreGaussian modes of radial and azimuthal orders $(n, l)$. Besides, as their Gaussian limit, by virtue of (4.2), also the $\phi_{n, l, E}^{(N)}$ 's can be generated from the fundamental " $2 \mathrm{D}$ Lorentz beam" $\phi_{0,0, E}^{(N)}(\rho, \vartheta) \propto\left(1+\frac{\rho^{2}}{N}\right)^{-N-\frac{1}{2}}$ through the same repeated derivative scheme [22, 23]:

$$
\phi_{n, l, E}^{(N)}(\rho, \vartheta) \propto\left(\frac{\partial}{\partial \omega^{*}}\right)^{l}\left(\frac{\partial}{\partial \omega}\right)^{n+l}\left(1+\frac{\rho^{2}}{N}\right)^{-N-\frac{1}{2}}
$$

with $\rho^{2}=\omega \omega^{*}$ and $\omega \equiv \xi+i \eta$. 
One can also argue that the $\phi_{n, l, E}^{(N)}$ 's should relate to the circular beams, introduced in [12], as the 2D rotationally symmetric counterpart of the Cartesian beams introduced in [4] and [5], whose relation with the $\varphi_{n, E}^{(N)}(\xi)$ has been clarified in Sect. 3.3. Such circular beams have Whittaker's first function $M_{\kappa, \mu}$ as their central part.

However, let us say that this seems to be only a pure speculation since optical fields shaping as (4.3) appear hardly implementable. Primarily, we note that, to our knowledge, rotationally symmetric optical fields having a radial trend as that of a Lorentzian, i.e. of the type $\frac{1}{1+\rho^{2}}$, have not been experimentally observed.

Finally, following the suggestion put forward in [14], we may say that a fractional order version of the EHL beams can be worked out through the obvious definition

$$
\varphi_{\nu, E}^{(N)}(\xi) \propto\left(-\frac{d}{d \xi}\right)^{\nu}\left(1+\frac{\xi^{2}}{N}\right)^{-N}
$$

with $\nu$ non-negative real.

Then, going through the explicit calculation on account of that the aforementioned derivative rule for the Fourier transform applies also to the fractional order derivative, if the latter is defined according to the Riemann-Liouville integral, namely:

$$
{ }_{-\infty} D_{x}^{\nu} f(x)=\frac{1}{\Gamma(m-\nu)} \frac{d^{m}}{d x^{m}} \int_{-\infty}^{x}\left(x-x^{\prime}\right)^{m-\nu-1} f\left(x^{\prime}\right) d x^{\prime},
$$

where $m$ is the nearest integer exceeding $\nu$, we end up with a rather obvious real-order form of the $\varphi_{n, E}^{(N)}$ 's in (2.15), involving of course the expression of the $H_{n}^{(N)}$ in terms of the Gauss hypergeometric series ${ }_{2} F_{1}$ with $n$ replaced by $\nu$ (or, equivalently that of the Gegenbauer function), i.e.

$$
\varphi_{\nu, E}^{(N)}(\xi) \propto \frac{\Gamma(\nu+2 N)}{N^{\frac{\nu}{2}} \Gamma(2 N)} \frac{(\xi /|\xi|)^{\nu}}{\left(1+\frac{\xi^{2}}{N}\right)^{N+\frac{\nu}{2}}}{ }_{2} F_{1}\left(-\frac{\nu}{2}, N+\frac{\nu}{2} ; N+\frac{1}{2} ; \frac{1}{1+\frac{\xi^{2}}{N}}\right) .
$$

Evidently it is no longer real in contrast to the $\varphi_{n, E}^{(N)}$, s, and also has a phase-discontinuity of $\pi$ at $\xi=0$.

We conclude evidencing a further optical interpretation of the RHPs. We may see in fact that they convey the order-dependent part of the transverse amplitude of the guided modes in a hyperbolic secant (HS) planar waveguide $[26]^{6}$. Such amplitudes can be written as

$$
\psi_{n}^{(s)}(u) \propto\left(\frac{1}{1+u^{2}}\right)^{\frac{s}{2}} H_{n}^{\left(s-n+\frac{1}{2}\right)}\left(u \sqrt{s-n+\frac{1}{2}}\right),
$$

where $u$ is the hyperbolic coordinate: $u(\xi)=\sinh (\alpha \xi)$. The parameter $\alpha$ and $s$ define the details of the index profile, and hence of the propagation constants $\beta_{n}$ of the modes [26]. Since in general $s-n+\frac{1}{2}$ is not an integer number, the RHPs involved in (4.7) demand for non integer values of the inherent parameter $N$.

The paraxial and non-paraxial propagation of light in a planar waveguide with a HS refractive index profile has been the object of relatively recent studies [27, 28].

\footnotetext{
${ }^{6}$ See worked problems at end of Sec. 23. The formal similarity between the eigenvalue equation for the guided modes in a waveguide with a hyperbolic secant refractive index profile and the time-independent Schrödinger equation for a particle in a hyperbolic secant potential distribution is evident.
} 


\section{Acknowledgment}

The authors wish to thank an anonymous referee for interesting comments.

\section{Appendix}

The determination of the normalization factor $\mathcal{N}_{n, N}^{(E)}$ entering the expression of the $\varphi_{n, E}^{(N)}$,s amounts to evaluating the overlap integral

$$
I_{n, m}^{(N)}=\int_{-\infty}^{\infty} \varphi_{n, E}^{(N)}(\xi)\left[\varphi_{m, E}^{(N)}(\xi)\right]^{*} d \xi,
$$

which evidently differs from zero only for $n+m=$ even, due to the parity properties of the $\varphi_{n, E}^{(N)}$ 's.

Resorting to the Parseval theorem and to the possibility of representing the $\varphi_{n, E}^{(N)}$, s as $\varphi_{n, E}^{(N)}(\xi) \propto \widehat{\mathcal{F}}^{-1}(-i \kappa)^{n} \widehat{\mathcal{F}}\left(1+\frac{\xi^{2}}{N}\right)^{-N}$, we see that the above integral is equivalent to

$$
I_{n, m}^{(N)}=\left|\mathcal{N}_{n, N}^{(E)}\right|^{2} \int_{-\infty}^{\infty}(-i \kappa)^{n}(i \kappa)^{m}\left|\widehat{\mathcal{F}}\left(1+\frac{\xi^{2}}{N}\right)^{-N}\right|^{2} d \kappa
$$

Since, as noted in the footnote $3, \widehat{\mathcal{F}}\left(1+\frac{\xi^{2}}{N}\right)^{-N}=\frac{\sqrt{\pi}}{2^{N-\frac{3}{2}}} \frac{N^{N-\frac{1}{2}}}{\Gamma(N)}|\kappa|^{N-1} W_{0, N-\frac{1}{2}}(2 \sqrt{N}|\kappa|)$, with $W_{v, \mu}$ denoting Whittaker's second function, we are confronted with the expression

$$
I_{n, m}^{(N)}=(-)^{\frac{n-m}{2}} \frac{\pi \mathcal{N}_{n, N}^{(E)} 2}{2^{2(N-1)}} \frac{N^{N}}{\Gamma(N)^{2}} \int_{-\infty}^{\infty} \kappa^{2 N+n+m-2}\left[W_{0, N-\frac{1}{2}}(2 \sqrt{N} \kappa)\right]^{2} d \kappa, \quad n+m=\text { even }
$$

which evaluates to (see [24] for the involved integral)

$$
I_{n, m}^{(N)}=(-)^{\frac{n-m}{2}} \frac{\pi\left|\mathcal{N}_{n, N}^{(E)}\right|^{2}}{2^{2 N-1} N^{\frac{n+m-1}{2}}} \frac{\Gamma\left(N+\frac{n+m}{2}\right) \Gamma\left(\frac{n+m+1}{2}\right) \Gamma\left(2 N+\frac{n+m-1}{2}\right)}{\Gamma(N)^{2} \Gamma\left(N+\frac{n+m+1}{2}\right)} .
$$

The normalization factor $\mathcal{N}_{n, N}^{(E)}$ of our concern writes therefore in the form

$$
\mathcal{N}_{n, N}^{(E)}=2^{N-\frac{1}{2}} \Gamma(N)\left[\frac{N^{n-\frac{1}{2}} \Gamma\left(N+n+\frac{1}{2}\right)}{\sqrt{\pi} \Gamma(N+n) \Gamma\left(2 N+n-\frac{1}{2}\right) \Gamma\left(n+\frac{1}{2}\right)}\right]^{\frac{1}{2}} .
$$

We see that for $N \rightarrow \infty$ the above turns into

$$
\mathcal{N}_{n, N}^{(E)} \underset{N \rightarrow \infty}{\rightarrow} \frac{1}{\sqrt{2^{n-\frac{1}{2}} \Gamma\left(n+\frac{1}{2}\right)}}
$$

which is indeed the normalization factor appropriate for the elegant HG functions $H_{n}(\xi) e^{-\xi^{2}}$.

Some other interesting features of the $\varphi_{n, E}^{(N)}$, s could be investigated. In particular, we consider here the spatial and spatial frequency variances $\left\langle\xi^{2}\right\rangle_{n, N}$ and $\left\langle-\frac{\partial^{2}}{\partial \xi^{2}}\right\rangle_{n, N}$. So, in order to evaluate the former, we note that

$$
\begin{aligned}
& \left\langle\xi^{2}\right\rangle_{n, N} \equiv \sigma_{n, N}^{2}=\int_{-\infty}^{\infty} \varphi_{n, E}^{(N)}(\xi) \xi^{2}\left[\varphi_{n, E}^{(N)}(\xi)\right]^{*} d \xi \\
& =\left|\mathcal{N}_{n, N}^{(E)}\right|^{2} \int_{-\infty}^{\infty}\left|\frac{\partial}{\partial \kappa}(-i \kappa)^{n} \widehat{\mathcal{F}}\left(1+\frac{\xi^{2}}{N}\right)^{-N}\right|^{2} d \kappa
\end{aligned}
$$


which after some algebra yields for the spatial variance at the plane $\zeta=0$ the expression

$$
\sigma_{n, N}^{2}=\sigma_{n, \infty}^{2} \frac{1+\frac{1-3 n+2 n^{2}}{(4 n-1) N}}{\left(1+\frac{n-1}{N}\right)\left(1+\frac{2 n-3}{4 N}\right)},
$$

where

$$
\sigma_{n, \infty}^{2}=\frac{1}{4} \frac{4 n-1}{2 n-1}
$$

is the limit of the above expression for $N \rightarrow \infty$ and in fact reproduces the expression for the spatial variance of the EHG modes at the input plane (see, for instance, [25]).

Similarly, as to the spatial frequency variance $\left\langle-\frac{\partial^{2}}{\partial \xi^{2}}\right\rangle_{n, N}$, on account of that

$$
\begin{gathered}
\left\langle-\frac{\partial^{2}}{\partial \xi^{2}}\right\rangle_{n, N} \equiv \widetilde{\sigma}_{n, N}^{2}=-\int_{-\infty}^{\infty} \varphi_{n, E}^{(N)}(\xi) \frac{\partial^{2}}{\partial \xi^{2}}\left[\varphi_{n, E}^{(N)}(\xi)\right]^{*} d \xi \\
=\int_{-\infty}^{\infty}\left|\frac{\partial}{\partial \xi} \varphi_{n, E}^{(N)}(\xi)\right|^{2} d \xi=\frac{\left|\mathcal{N}_{n, N}^{(E)}\right|^{2}}{\left|\mathcal{N}_{n+1, N}^{(E)}\right|^{2}},
\end{gathered}
$$

we obtain

$$
\tilde{\sigma}_{n, N}^{2}=\widetilde{\sigma}_{n, \infty}^{2} \frac{\left(1+\frac{n}{N}\right)\left(1+\frac{2 n-1}{4 N}\right)}{\left(1+\frac{2 n+1}{2 N}\right)},
$$

where $\widetilde{\sigma}_{n, \infty}^{2}$ reproduces the expression pertaining to the EHG modes [25]:

$$
\widetilde{\sigma}_{n, \infty}^{2}=2 n+1 .
$$

The specific values of $\sigma_{n, N}^{2}$ and $\widetilde{\sigma}_{n, N}^{2}$ for some $n$ 's and $N$ 's are reported in Table 1 along with the values for the limiting EHG beams. In particular, the values relative to $N=1$ support the considerations developed in Sec. 3.1 as to the behavior of the $N=1$ higher order EHL beams, graphically conveyed by the contourplots in Fig. 3.2.

Table 1 Spatial and spatial frequency variances $\sigma_{n, N}^{2}$ and $\widetilde{\sigma}_{n, N}^{2}$

\begin{tabular}{lllllllll}
\hline$n$ & $\sigma_{n, 1}^{2}$ & $\tilde{\sigma}_{n, 1}^{2}$ & $\sigma_{n, 5}^{2}$ & $\tilde{\sigma}_{n, 5}^{2}$ & $\sigma_{n, 15}^{2}$ & $\tilde{\sigma}_{n, 15}^{2}$ & $\sigma_{n, \infty}^{2}$ & $\tilde{\sigma}_{n, \infty}^{2}$ \\
\hline 0 & 1 & 0.5 & 0.294 & 0.864 & 0.263 & 0.952 & 0.25 & 1 \\
1 & 1 & 3 & 0.789 & 2.908 & 0.763 & 2.958 & 0.75 & 3 \\
2 & 0.333 & 7.5 & 0.503 & 5.367 & 0.553 & 5.1 & 0.583 & 5 \\
3 & 0.2 & 14 & 0.404 & 8.235 & 0.49 & 7.378 & 0.55 & 7 \\
4 & 0.143 & 22.5 & 0.343 & 11.511 & 0.451 & 9.792 & 0.536 & 9 \\
5 & 0.111 & 33 & 0.299 & 15.19 & 0.42 & 12.341 & 0.528 & 11
\end{tabular}

We see that in general, for a fixed $N, \sigma_{n, N}^{2}$ decreases with increasing $n$ more or less rapidly according to the value of $N$ (with being $\sigma_{n, \infty}^{2} \underset{n \rightarrow \infty}{\rightarrow} 0.5$ ), whilst correspondingly $\widetilde{\sigma}_{n, N}^{2}$ increases. As earlier noted, this is due to the factor $\left(1+\frac{\xi^{2}}{N}\right)^{-n-N}$ which modulates the polynomial $H_{n}^{(N)}$, and may therefore induce to consider alternatively the perhaps more convenient "transversal" sets of functions $\varphi_{n, E}^{(N-n)}(\xi)$ for $N>n$, as suggested in the concluding notes.

We also see that, as expected according to the uncertainty principle, the product $\sigma_{n, N} \widetilde{\sigma}_{n, N}$ for all $n$ and $N$ is greater than $\sigma_{0, \infty} \widetilde{\sigma}_{0, \infty}=\frac{1}{2}$, the value pertaining to the 
fundamental Gaussian function which is indeed the waveform that minimizes the uncertainty product. On the other hand, for $n \geq 2$ the aforementioned product increases with $n$ less quickly than that for the limiting EHG functions, remaining lower than it and approaching it for $N \rightarrow \infty: \sigma_{n, N} \widetilde{\sigma}_{n, N} \underset{N \rightarrow \infty}{\longrightarrow} \sigma_{n, \infty} \widetilde{\sigma}_{n, \infty} \underset{n \rightarrow \infty}{\rightarrow} \infty$.

In this connection, it may be interesting to recall that the uncertainty product for the SHG functions, having the same characteristic width, i.e. $\frac{1}{\sqrt{2^{n-\frac{1}{2}} n ! \sqrt{\pi}}} H_{n}(\sqrt{2} \xi) e^{-\xi^{2}}$, scales as $\frac{1}{2}(2 n+1)$, and so for $n \geq 2$ increases with $n$ more quickly than that for the EHG functions $\varphi_{n, E}^{(\infty)}(\xi)$. This is a well known behavior, justified by the fact that, as noted in [25], the shape of the EHG functions remains much closer to Gaussian in form than that of the SHG functions. In fact, the SHG functions (as their Fourier transforms) distribute the power through an $n$-dependent number of peaks, specifically $n+1$, whose height increases with increasing distance from $\xi=0(\kappa=0$, respectively). In contrast, the EHG functions concentrate the power into a few peaks about $\xi=0$. Correspondingly, the relevant Fourier transforms $\widetilde{\varphi}_{n, E}^{(\infty)}(\kappa)=\frac{(-i \kappa)^{n}}{\sqrt{2^{n+\frac{1}{2}} \Gamma\left(n+\frac{1}{2}\right)}} e^{-\frac{\kappa^{2}}{4}}$, for any value $n \neq 0$, display only two symmetrically placed intensity peaks whose separation increases with $n$ as $2 \sqrt{2 n}$, but in such a way that the spatial frequency variances of the EHG and SHG functions come to be equal.

In turn, the EHL functions $\varphi_{n, E}^{(N)}(\xi)$ convey the power even more strongly than the corresponding EHG functions in a few peaks about $\xi=0$. Thus, for instance, for any moderate value of $n$ greater or equal to $2(2 \leq n \leq 8)$ one may basically detect three central intensity peaks of appreciable height for $n$ even, and two symmetrically placed peaks for $n$ odd. See the plots in Fig. 2.1. Correspondingly, the Fourier transforms $\widetilde{\varphi}_{n, E}^{(N)}(\kappa)$, for $n \neq 0$, have only two intensity peaks symmetrically placed about $\kappa=0$. The separation of these two peaks increases with $n$ at a rate which depends on $N$ and attains its maximum value (of $2 n$ ) for $N=1$. See the plots in Figs. 3.1c-d).

As a result, for $n \geq 2$ the space-bandwidth product for the EHL functions increases with $n$ less quickly than that for the EHG functions, the latter being recovered for $N \rightarrow \infty$.

As is a well known, the space-bandwidth product (properly normalized) is often taken as a "measure" of the quality of an optical beam, higher values of it signifying poorer quality [29]. However, since the concept of beam quality is neither unique nor universal, definite properties of interest for specific applications deserves further parameters being adopted as estimators of the related beam quality. Thus, for instance, as earlier noted, if paraxiality is a property of concern, more qualifying estimators, like those introduced in [19] and [20], would be more suitable to "measure" the associated beam quality. Also, the significance of the space-bandwidth product as an estimator of the beam quality definitely relates to the applicability of the paraxial approach to beam propagation.

Finally, we note that, as a further distinguishing feature of the Lorentz beams with respect to the Gaussian ones, the relevant even intensity moments $\left\langle\xi^{2 m}\right\rangle$ diverge for any $2 m \geq 4 N+2 n$.

\section{References}

[1] O. El Gawhary and S. Severini, Lorentz beams and symmetry properties in paraxial optics, J. Opt. A. Pure Appl. Opt. 8, 409-414 (2006). 
[2] W.P. Dumke, The angular beam divergence in double-heterojunction lasers with very thin active regions, IEEE J. Quantum Electron. 11, 400-402 (1975)

[3] A. Naqwi and F. Durst, Focusing of diode laser beams: a simple mathematical model, Appl. Opt. 29, 1780-1785 (1990)

[4] M.A. Bandres and J.C. Gutiérrez-Vega, Cartesian beams, Opt. Lett. 32, 3459-3461 (2007)

[5] A. Torre, A note on the general solution of the paraxial wave equation: a Lie algebra view, J. Opt. A: Pure Appl. Opt. 10, (2008) 055006

[6] V. Aldaya, J. Bisquert and J. Navarro-Salas, The quantum relativistic harmonic oscillator: generalized Hermite polynomials, Phys. Lett. A 156, 381-385 (1991)

[7] B. Nagel, The relativistic Hermite polynomial is a Gegenbauer polynomial, J. Math. Phys. 35, 1549-1554 (1994)

[8] G. Dattoli, S. Lorenzutta, G. Maino and A. Torre, The generating function method and properties of the relativistic Hermite polynomials, Nuovo Cim. 113 B, 553-560 (1998)

[9] Mourad E.H. Ismail, Relativistic orthogonal polynomials are Jacobi polynomials, J. Phys. A: Math. Gen. 29, 3199-3202 (1996)

[10] F. Brackx, N. De Schepper and F. Sommen, The Clifford-Gegenbauer polynomials and the associated continuous wavelet transform, Int. Transf. \& Spec. Funct. 15, 387-404 (2004)

[11] P. Natalini, The relativistic Laguerre polynomials, Rend. Matematica, Ser. VII, 16, 299-313 (1996)

[12] M.A. Bandres and J.C. Gutiérrez-Vega, Circular beams, Opt. Lett. 33, 177-179 (2008)

[13] E.G. Kalnins and W. Miller Jr., Lie theory and separation of variables. 5 . The equation $i U_{t}+U_{x x}=0$ and $i U_{t}+U_{x x}-c / x^{2} U=0$, J. Math. Phys. 15, 1728-1737 (1974)

[14] J.C. Gutiérrez-Vega, Fractionalization of optical beams: I. Planar analysis, Opt. Lett. 32, 1521-1523 (2007).

[15] A. Erdélyi, W. Magnus, F. Oberhettinger and F.G. Tricomi, Higher Transcendental Functions, Vols. I and II, (MacGraw-Hill, New York, London and Toronto, 1953).

[16] N. Gurappa and P.K. Panigrahi, Equivalence of the Sutherland model to free particles on a circle, hep-th/9908127 (unpublished); N. Gurappa, P.K. Panigrahi, T. Shreecharan and S. Sree Ranjani, A novel method to solve familiar differential eqautions and its applications, in Frontiers of Fundamental Physics, vol. 4, ed. B.G. Sidharth and M.V. Altaisky (Kluwer/Plenum, New York, 2001), 269-278

[17] K.B. Wolf, Canonical transforms.I. Complex linear transforms, J. Math. Phys. 15, 1295-1301 (1975), and refs. \#14 and 18 therein. 
[18] O. El Gawhary and S. Severini, Lorentz beams as a basis for a new class of rectangularly symmetric optical fields, Opt. Comm. 269, 274-284 (2007)

[19] P. Vaveliuk, B. Ruiz and A. Lencina, Limits of the paraxial approximation in laser beams, Opt. Lett. 32, 927-929 (2007)

[20] O. El Gawhary and S. Severini, Degree of paraxiality for monochromatic light beams, Opt. Lett. 33, 1360-1362 (2008)

[21] H. Buccholz, The Confluent Hypergeometric Function (Springer-Verlag, Berlin, 1969).

[22] A. Wünsche, Generalized Gaussian beam solutions of paraxial optics and their connection to a hidden symmetry, J. Opt. Soc. Am. A 6, 1320-1329 (1989)

[23] J. Enderlein and F. Pampaloni, Unified operator approach for deriving HermiteGaussian and Laguerre-Gaussian laser modes, JOSA 21, 1553-1558 (2004)

[24] A. Erdélyi, W. Magnus, F. Oberhettinger and F.G. Tricomi, Tables of Integral Transforms, Vol. II (MacGraw-Hill, New York, London and Toronto, 1953), p. 409 \#41.

[25] S. Saghafi and C.J.R. Sheppard, The beam propagation factor for higher order Gaussian beams, Opt. Comm. 153, 207-210 (1998)

[26] L. Landau and E. Lifschitz, Quantum Mechanics (Pergamon Press, Oxford, 1965)

[27] J. Liñares and C. Gómez-Reino, Optical propagator in a graded-index medium with a hyperbolic secant refractive-index profile, Appl. Opt. 33, 3427-3431 (1994)

[28] C. Gómez-Reino, M.V. Perez, C. Bao, M.T. Flore-Arias, S. Vidal and S. Fernandez De Avila, Non-paraxial diffraction-free propagation of light in a graded-index planar waveguide with a hyperbolic secant refractive index profile, Jpn. J. Appl. Phys. 39, 1463-1467 (2000)

[29] http://www.stanford.edu/ siegman/beam_quality_tutorial_osa.pdf 
[18] O. El Gawhary and S. Severini, Lorentz beams as a basis for a new class of rectangularly symmetric optical fields, Opt. Comm. 269, 274-284 (2007)

[19] P. Vaveliuk, B. Ruiz and A. Lencina, Limits of the paraxial approximation in laser beams, Opt. Lett. 32, 927-929 (2007)

[20] O. El Gawhary and S. Severini, Degree of paraxiality for monochromatic light beams, Opt. Lett. 33, 1360-1362 (2008)

[21] H. Buccholz, The Confluent Hypergeometric Function (Springer-Verlag, Berlin, 1969).

[22] A. Wünsche, Generalized Gaussian beam solutions of paraxial optics and their connection to a hidden symmetry, J. Opt. Soc. Am. A 6, 1320-1329 (1989)

[23] J. Enderlein and F. Pampaloni, Unified operator approach for deriving HermiteGaussian and Laguerre-Gaussian laser modes, JOSA 21, 1553-1558 (2004)

[24] A. Erdélyi, W. Magnus, F. Oberhettinger and F.G. Tricomi, Tables of Integral Transforms, Vol. II (MacGraw-Hill, New York, London and Toronto, 1953), p. 409 \#41.

[25] S. Saghafi and C.J.R. Sheppard, The beam propagation factor for higher order Gaussian beams, Opt. Comm. 153, 207-210 (1998)

[26] L. Landau and E. Lifschitz, Quantum Mechanics (Pergamon Press, Oxford, 1965)

[27] J. Liñares and C. Gómez-Reino, Optical propagator in a graded-index medium with a hyperbolic secant refractive-index profile, Appl. Opt. 33, 3427-3431 (1994)

[28] C. Gómez-Reino, M.V. Perez, C. Bao, M.T. Flore-Arias, S. Vidal and S. Fernandez De Avila, Non-paraxial diffraction-free propagation of light in a graded-index planar waveguide with a hyperbolic secant refractive index profile, Jpn. J. Appl. Phys. 39, 1463-1467 (2000)

[29] http://www.stanford.edu/ siegman/beam_quality_tutorial_osa.pdf 